\title{
M 31 circum-nuclear region: A molecular survey with the IRAM interferometer ${ }^{\star}$
}

\author{
Julien Dassa-Terrier $^{1}$, Anne-Laure Melchior ${ }^{1}$, and Françoise Combes ${ }^{1,2}$ \\ 1 LERMA, Observatoire de Paris, PSL Research University, CNRS, Sorbonne Universités, UPMC Univ. Paris 06, 75014 Paris, France \\ e-mail: julien.dassa-terrier@obspm.fr,a.l.melchior@obspm.fr,francoise.combes@obspm.fr \\ 2 Collège de France, 11, Place Marcelin Berthelot, 75005 Paris, France
}

Received 10 August 2018 / Accepted 13 February 2019

\begin{abstract}
We analysed molecular observations performed at IRAM interferometer in $\mathrm{CO}(1-0)$ of the circum-nuclear region (within $250 \mathrm{pc}$ ) of Andromeda with $2.9^{\prime \prime}=11 \mathrm{pc}$ resolution. We detected 12 molecular clumps in this region, corresponding to a total molecular mass of $(8.4 \pm 0.4) \times 10^{4} M_{\odot}$. These clumps follow Larson's mass-size relation, but lie well above the velocity-size relation. We discuss the possibility that these clumps are probably not virialised, but are transient agglomerations of smaller entities that might be virialised. Three of these clumps have been detected in $\mathrm{CO}(2-1)$ in a previous work, and we find a temperature line ratio below 0.5 in this work. With a radiative transfer analysis, we show that this gas is in non-local thermal equilibrium with a low excitation temperature $\left(T_{\text {ex }}=5-9 \mathrm{~K}\right)$. We find a surface beam filling factor of order $5 \%$ and a gas density in the range $60-650 \mathrm{~cm}^{-3}$, which is well below the critical density. With a gas-to-stellar mass fraction of $4 \times 10^{-4}$ and dust-to-gas ratio of 0.01 , this quiescent region has exhausted its gas budget. Its spectral energy distribution is compatible with passive templates assembled from elliptical galaxies. While weak dust emission is present in the region, we show that no star formation is present and support the previous results that the dust is heated by the old and intermediate stellar population. We study the possibility that this region lies formally in the low-density part of the Kennicutt-Schmidt law in a regime where the star formation rate estimators are not completely reliable. We confirm the quiescence of the inner part of this galaxy known to lie on the green valley.
\end{abstract}

Key words. galaxies: individual: M 31 - galaxies: kinematics and dynamics - submillimeter: ISM - molecular data

\section{Introduction}

The evolution of the gas content and star formation activity in the central kiloparsec of galaxies is key to understanding the coupling of black hole evolution with the rest of the galaxy. Aside from the activity of the central engine probably powered by mass accretion (e.g. Lynden-Bell 1969; Urry \& Padovani 1995), the properties of the host galaxy are directly impacted by this so-called AGN feedback (Fabian 2012, and references therein). The scaling relations between the supermassive black hole mass and bulge mass and the velocity dispersion of the host (e.g. Ferrarese \& Merritt 2000; Tremaine et al. 2002; Marconi \& Hunt 2003; Gültekin et al. 2009; Kormendy \& Ho 2013; McConnell \& Ma 2013) suggest a close connection between supermassive black holes and their hosts (e.g. Silk \& Rees 1998; Di Matteo et al. 2005; King \& Pounds 2015). The growth of supermassive black holes over cosmic time is probably dominated by external gas accretion (e.g. Soltan 1982; Croton et al. 2006). Indeed, galaxy mergers (e.g. Barnes \& Hernquist 1991; Springel et al. 2005; Hopkins et al. 2006) or galactic bars (e.g. Pfenniger \& Norman 1990; Begelman et al. 2006; Hopkins \& Quataert 2010) may efficiently transport gas towards the galactic nucleus through gravitational torques (e.g. García-Burillo et al. 2005) over a few dynamical times.

^ The reduced spectra are only available at the CDS via anonymous ftp to cdsarc.u-strasbg.fr (130.79.128.5) or via http: //cdsarc.u-strasbg.fr/viz-bin/qcat?]/A+A/625/A148
While the total AGN activity is correlated with the global star formation rate (SFR) as a function of cosmic time (e.g. Heckman et al. 2004; Heckman \& Best 2014), SFR has been mostly quenched in the local Universe (e.g. Belfiore et al. 2016) while the activity of central black holes has been much reduced (e.g. Sijacki et al. 2015). The mechanisms responsible for this quenching are currently being investigated. Relying on simulations, Bower et al. (2017) have shown that these red and blue sequences of galaxies result from a competition between star formation-driven outflows and gas accretion onto the supermassive black hole at the centre of a galaxy. Belfiore et al. (2016) argued that the quenching occurred inside out and that the star formation first stopped in the central region. However, these inside-out mechanisms are probably not universal because in dense environments, such as groups or clusters of galaxies, the quenching could also occur outside-in. Peng et al. (2015) argued that the strangulation mechanism in which the supply of cold gas to the galaxy is halted is the main mechanism responsible for quenching star formation in local galaxies with $M_{*}<$ $10^{11} M_{\odot}$. In the GAs Stripping Phenomena in galaxies with MUSE (GASP) survey, Poggianti et al. (2017) have explored in the optical more than 100 galaxies in different environments with the idea to get better constraints on both types of scenarios. Gullieuszik et al. (2017) have shown that gas is stripped out in JO204 a jellyfish galaxy in A957 and the star formation activity is reduced in the outer part. With a study based on local galaxies, Fluetsch et al. (2019) have argued that AGN-driven outflows are likely capable of clearing and quenching the central region of galaxies. 
Table 1. Positions of the 4 fields observed at IRAM-PdB in 2012.

\begin{tabular}{lccccc}
\hline \hline Field & RA & Dec & Visibilities & Beam & Pixels \\
\hline 1 & $00^{\mathrm{h}} 42^{\mathrm{m}} 44.39^{\mathrm{s}}$ & $+41^{\circ} 16^{\prime} 08.3^{\prime \prime}$ & 4473 & $3.37^{\prime \prime} \times 2.45^{\prime \prime}$ & $0.61^{\prime \prime} \times 0.61^{\prime \prime}$ \\
2 & $00^{\mathrm{h}} 42^{\mathrm{m}} 46.78^{\mathrm{s}}$ & $+41^{\circ} 16^{\prime} 12.3^{\prime \prime}$ & 4432 & $3.37^{\prime \prime} \times 2.45^{\prime \prime}$ & $0.61^{\prime \prime} \times 0.61^{\prime \prime}$ \\
3 & $00^{\mathrm{h}} 42^{\mathrm{m}} 45.27^{\mathrm{s}}$ & $+41^{\circ} 15^{\prime} 54.3^{\prime \prime}$ & 4743 & $4.53^{\prime \prime} \times 2.76^{\prime \prime}$ & $0.74^{\prime \prime} \times 0.74^{\prime \prime}$ \\
4 & $00^{\mathrm{h}} 42^{\mathrm{m}} 43.50^{\mathrm{s}}$ & $+41^{\circ} 15^{\prime} 36.3^{\prime \prime}$ & 3131 & $4.53^{\prime \prime} \times 2.76^{\prime \prime}$ & $0.74^{\prime \prime} \times 0.74^{\prime \prime}$ \\
\hline
\end{tabular}

Notes. The phase centre of the observation was $00^{\mathrm{h}} 42^{\mathrm{m}} 44.99^{\mathrm{s}},+41^{\circ} 15^{\prime} 57.8^{\prime \prime}$. We provide the $\mathrm{J} 2000.0$ equatorial coordinates of the centre of each field, the number of visibilities acquired per field, and the corresponding beam and pixel size (square). The sampling of the 4th field is worse than for the others, which explains a larger level of noise.

With a stellar mass of $5 \times 10^{10} M_{\odot}$ (Viaene et al. 2014), Andromeda belongs to the transition regime between the active blue-sequence galaxies and passive red-sequence galaxies (e.g. Bower et al. 2017; Baldry et al. 2006), which happens around the stellar mass of $3 \times 10^{10} M_{\odot}$ (e.g. Kauffmann et al. 2003). This galaxy is a prototype galaxy from the Local Group where the star formation has been quenched in the central part. Andromeda hosts both very little gas and very little star formation, while the black hole is basically quiet and has some murmurs (Li et al. 2011). In a previous study about M31 nucleus, Melchior \& Combes (2017) have shown that there is no gas within the sphere of influence of the black hole. Indeed, the gas has been exhausted. Most scenarios of the past of evolution of Andromeda reproduce the large scale distribution and show evidence of a past activity rich in collisions (Ibata et al. 2001, 2014; Thilker et al. 2004; Gordon et al. 2006; McConnachie et al. 2009; Miki et al. 2016; Hammer et al. 2018). However, the exact mechanism quenching the activity in the central kiloparsec is still unknown (Tenjes et al. 2017). Block et al. (2006) proposed a frontal collision with M 32, which could account for the two ring structures observed in the dust distribution. Melchior \& Combes $(2011,2016)$ showed the presence of gas along the minor axis and support the scenario of the superimposition of an inner $1 \mathrm{kpc}$ ring with an inner disc. Melchior \& Combes (2013) estimated a minimum total mass of $4.2 \times 10^{4} M_{\odot}$ of molecular gas within a (projected) distance to the black hole of $100 \mathrm{pc}$. This is several orders of magnitude smaller than the molecular gas present in the central molecular zone of the Milky Way (Pierce-Price et al. 2000; Molinari et al. 2011). In the Galaxy, while large amounts of dense gas are present in the central region, Kruijssen et al. (2014) discussed the different processes that combine to inhibit star formation, which was observed to be a factor ten times weaker than expected (e.g. Leroy et al. 2008).

In this article, we analyse new molecular gas observations obtained with IRAM Plateau-de-Bure interferometer achieving a $11 \mathrm{pc}$ resolution. This resolution is well below the typical size at which a tight correlation is observed between SFR and gas density (K-S law). We study the properties of this gas and how it relates with the star formation activity in this region.

In Sect. 2, we present the analysis of the data cube, enabling an automatic selection of 12 molecular clouds. In Sect. 3, we discuss the properties of these clumps. In Sect. 4, we discuss how this detection of molecular clouds in the equivalent of the central molecular zone correlates with information available on star formation activity.

\section{Data analysis}

We describe the identification of molecular clumps in the IRAM-PdB data cube. In Sect. 2.1, we describe the IRAM-PdB observations and data reduction. In Sect. 2.2, we select the core of significant clumps above a given threshold and discuss their significance. In Sect. 2.3, we compute the 3D size, velocity dispersion, and total flux of these core clumps following the method proposed by Rosolowsky \& Leroy (2006). In Sect. 2.4, we discuss the velocity distribution of the detections and proceed to clean side lobes. In Sect. 2.5, we apply the principal component analysis (PCA) statistical method to discriminate reliable candidates using the descriptive parameters of the previous subsections. A final check is performed with a visual inspection of each selected clump. Last, in Sect. 2.6, we sum up our procedure and present our final selection.

\subsection{Observations and data reduction}

We present an analysis of an IRAM-PdB interferometer mosaic of four fields covering the centre of Andromeda and a field of view of about 2 arcmin off-centred to the south-east. As described in Melchior \& Combes (2017), the initial motivation of these observations was to search for molecular gas next to the black hole, and the region corresponding to the sphere of influence of M 31's black hole, i.e. $R_{\text {SOI }} \sim 14 \mathrm{pc}$, was explored. A 2 mJy signal with a linewidth of $1000 \mathrm{~km} \mathrm{~s}^{-1}$ was expected by Chang et al. (2007), but was excluded at a $9 \sigma$ level. We only detected a small $2000 M_{\odot}$ clump, most probably lying outside the sphere of influence of the black hole, which is seen in projection. In this article, we extend this first exploration to the whole data cube that covers the equivalent of the central molecular zone for the Milky Way (Morris \& Serabyn 1996; Oka et al. 1996). The observations were performed in 2012 with the five antenna configuration with the WideX correlator. It covers a wide velocity band $[-3000,6000] \mathrm{km} \mathrm{s}^{-1}$ and has a velocity resolution of $\delta v=5.07 \mathrm{~km} \mathrm{~s}^{-1}$ and a pixel size of $0.61^{\prime \prime}$. Four fields were observed at the positions provided in Table 1 and integrated for about four hours. A standard reduction was performed with the GILDAS software and these fields were combined. We performed our analysis on the mosaic assembled with a beam of $3.37^{\prime \prime} \times 2.45^{\prime \prime}\left(\mathrm{PA}=70^{\circ}\right)$ and a pixel size of $0.61^{\prime \prime}$. We focus our work on the $[-600,0] \mathrm{km} \mathrm{s}^{-1}$ velocity band, binned in 119 channels.

A quick view of the data cube confirms the absence of large amount of gas in this region. However, there are numerous clumps of molecular gas with low velocity dispersions. In Fig. 1, we observe a non-Gaussian flux distribution typical of a data cube with a signal. We optimised a selection procedure to disentangle genuine clumps from noise. Given this configuration, we performed a one-iteration CLEAN procedure to correct for the primary beam. We do not have single dish observations in $\mathrm{CO}(1-0)$ of this region and therefore we cannot correct the observations from missing short-spacing flux. In addition, as the signals are not extended and relatively weak, it is not possible 

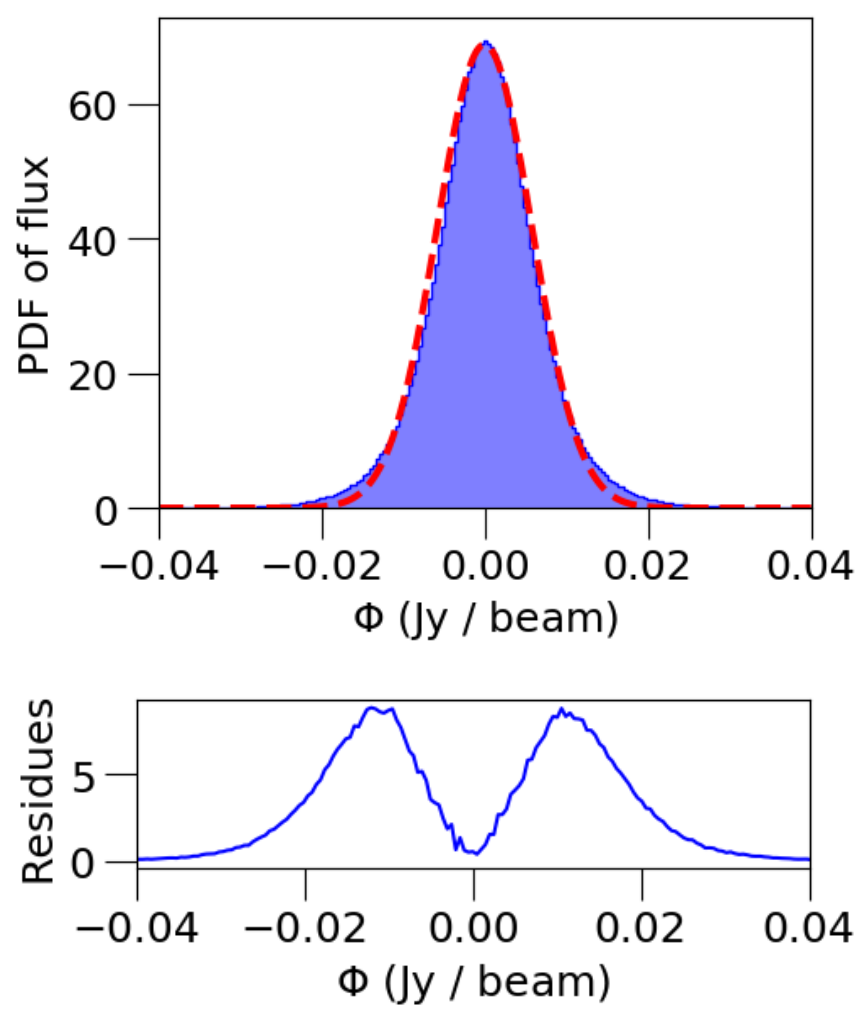

Fig. 1. Top: probability density function of the distribution of fluxes within the data cube and the associated Gaussian fit (red dashed curve). The value $\phi$ corresponds to the flux of each pixel (in the 3D data cube), and the spatial variation of the noise has not been corrected at this stage. Bottom: residues associated with the distribution and its Gaussian fit We observe that the distribution is not Gaussian, which we expect from a cube containing a signal. The residues are mostly symmetrical, showing that the signal is very faint and needs advanced detection methods.

to analyse the data cube with standard algorithms; for example the signal characteristics do not match the criteria stated in Rosolowsky \& Leroy (2006). Hence, we performed a basic signal detection in the data cube in an automatic way, and checked our results with careful visual inspections and classical methods (e.g. GILDAS).

\subsection{Detection of $3 \sigma$ peaks}

We relied on the first and second moments of each spectra with a $3 \sigma$ clip to get a best estimate of the mean flux $\langle\Phi\rangle(x, y)$ and rms noise level $\sigma_{\text {noise }}(x, y)$, where $(x, y)$ refers to the pixel position. We refer to these parameters simply as $\langle\Phi\rangle$ and $\sigma_{\text {noise }}$ hereafter. The noise level, of which a map is shown in Melchior \& Combes (2017), is at its lowest close to the black hole about $3.2 \mathrm{mJy}_{\text {beam }}^{-1}$ at the velocity resolution $\left(5.07 \mathrm{~km} \mathrm{~s}^{-1}\right)$, and increases towards the edges of the field of view (up to $13 \mathrm{mJy} \mathrm{beam}^{-1}$ ). In Appendix A, we derive $3 \sigma$ upper limit on the continuum level: about $15 \mu \mathrm{Jy}$ within $15^{\prime \prime}$ estimated on the whole bandwidth $[-3000,6000] \mathrm{km} \mathrm{s}^{-1}$.

We selected pixels whose spectrum exhibits more than two consecutive spectral channels with a flux excess or deficit of $3 \sigma_{\text {noise }}$. At this stage, we kept the negative signals to access the coherence of the detections with respect to noise. As detailed in Table 2, we detected 1045 pixels: 663 (resp. 382) with a positive (resp. negative) signal. As isolated pixels or aggregates of two pixels are most likely noise, we define clump candidates as groups of at least three adjacent pixels exhibiting more than two consecutive channels at $3 \sigma_{\text {noise }}$ from the mean level. In principle, this criteria was used to select the core of possible clumps, i.e. the central $3 \sigma$ pixels with high signal-to-noise ratio $(\mathrm{S} / \mathrm{N})$. We excluded 142 pixels ( 65 positive and 77 negative) with less than 3 adjacent pixels. We thus kept 598 (resp. 305) pixels gathered in 54 positive (resp. 47 negative) $3 \sigma$ clumps. Of the detected signals $33.8 \%$ are negative. Positive (resp. negative) $3 \sigma$ clumps have a mean flux of $766 \mathrm{mJy} \mathrm{beam}^{-1}$ (resp. $-313 \mathrm{mJy} \mathrm{beam}^{-1}$ ). Their average surface is of 11.1 pixels (compared to 6.5 for the negative $3 \sigma$ clumps). The number of pixels at half-power beam width (HPBW) is about 25 pixels, hence we only see the inner parts of the clumps. Given the relatively small number of pixels in the $3 \sigma$ clumps compared to 25 , we expect that the detections are close to the spatial resolution. Figure B.1 shows the mean velocity map of positive (left panel) and negative (right panel) $3 \sigma$ peak detections above $3 \sigma_{\text {noise }}$.

We estimate the peak $\mathrm{S} / \mathrm{N}\left(S N R_{\text {peak }}\right)$ associated with detected $3 \sigma$ clumps as the ratio of the peak flux $\Phi_{\text {peak }}$ of the $3 \sigma$ clump to the noise estimated for its spectra. We represent this $\mathrm{S} / \mathrm{N}$ distribution in Fig. 2. The average $S N R_{\text {peak }}$ for the positive (resp. negative) $3 \sigma$ clumps is 4.4 (resp. -3.7 ) with 28 positive (resp. 16 negative) $3 \sigma$ clumps showing a peak signal higher than $4 \sigma_{\text {noise }}$ (resp. lower than $-4 \sigma_{\text {noise }}$ ), and 3 positive $3 \sigma$ clumps with peak $\mathrm{S} / \mathrm{N}$ larger than 8 . The clumps we detected are thus significantly brighter than the level of noise, estimated with the negative clumps. Although the two sets behave differently, more investigations are required to select genuine molecular clouds with confidence. In the three next subsections, we calculate global properties, exclude side lobes, and apply PCA to make our final selection.

\subsection{Physical quantities derived from moment measurements}

In order to further characterise the detection of molecular clouds, we estimate the clump sizes, velocity dispersions, and total fluxes. Rosolowsky \& Leroy (2006) noted a strong correlation between the resolution and the measured size of clumps for those with a size close to the spatial resolution. This is probably the case in this work: most detected clumps are unresolved or close to the detection limit. We expect the clumps to have an elliptical shape similar to the beam. Hence, we adapted the Cloud PROPertieS (CPROPS) method proposed by Rosolowsky \& Leroy (2006) to optimise these measurements. We note that Rosolowsky \& Leroy (2006) showed that this extrapolating method is optimum for a peak $\mathrm{S} / \mathrm{N}$ larger than 10 , while our data host only 2 clumps as strong as this. However, these authors showed that the properties derived from interferometric data are underestimated with respect to single dish data, with a lost of order of $50 \%$ in the integrated luminosity as already discussed by Sheth et al. (2000).

We calculate the second moments of the major $x$-axis and minor $y$-axis (defined for each clump). A PCA, also performed with similar intent in Koda et al. (2006), is used to define these axes: $x$ and $y$ refer to the major and minor axes, respectively, hereafter. For each clump, we define an elliptical box with major and minor axes twice as big as the beam centred on the pixel with peak flux $\Phi_{\max }$. This intends to exclude surrounding regions with potential noise. We define a variable $\Phi_{\text {iso, }}$, which corresponds to the equal-intensity isophotes. We, then, studied the evolution of the size, velocity dispersion, and total flux as a function of the intensity values corresponding to each isophote, varying from $\Phi_{\max }$ to $2 \sigma_{\text {noise }}$. Up to 100 isophotes are then used for each clump. We chose 100 isophotes since our box size may contain up to 60 pixels with 3 to 6 channels. Each value of $\Phi_{\text {iso }}$ delimits a region over which we sum the following moments over all 
Table 2. Summary of the identification of cores process.

\begin{tabular}{|c|c|c|c|c|c|c|c|c|c|c|}
\hline & \multicolumn{3}{|c|}{ Number of pixels } & \multicolumn{3}{|c|}{ Core clumps } & \multicolumn{2}{|c|}{ Mean surface (pixels) } & \multicolumn{2}{|c|}{ Approx. mean flux $\left(\mathrm{mJy}\right.$ beam $\left.^{-1}\right)$} \\
\hline & Total & Positive & Negative & Total & Positive & Negative & Positive & Negative & Positive & Negative \\
\hline$\leq 2$ pixels & 142 & 65 & 77 & 102 & 47 & 55 & & & & \\
\hline$>2$ pixels & 903 & 598 & 305 & 101 & 54 & 47 & 11.1 & 5.6 & 766 & -313 \\
\hline
\end{tabular}

Notes. We select spectra with at least 2 consecutive channels deviating from $3 \sigma$ from the continuum level. We keep positive and negative signals at each stage in order to have an estimate of the noise level. We provide the number of pixels and the number of core clumps derived. Only core clumps with at least 3 adjacent pixels are kept for subsequent analysis. The approximated mean flux is the mean value of the sums of detected pixels fluxes of all core clumps.

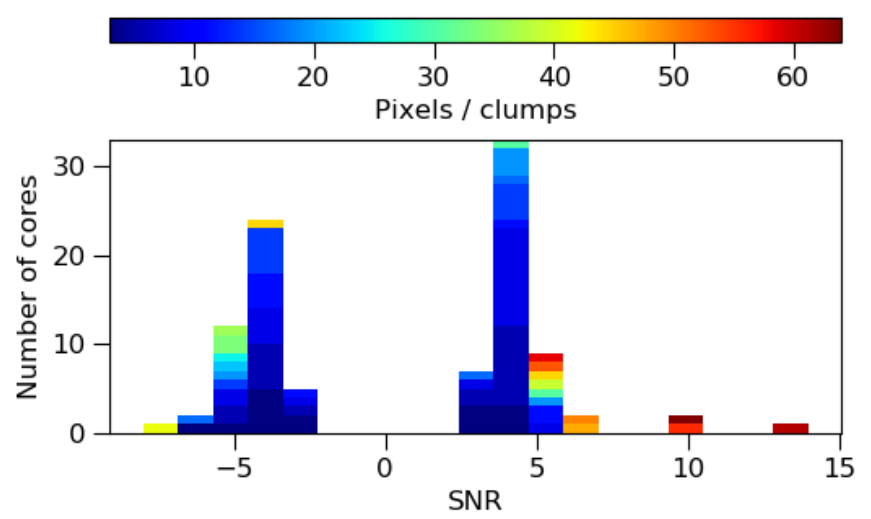

Fig. 2. Distribution of the $\mathrm{S} / \mathrm{N}$ based on the $3 \sigma$ peak flux $\left(S N R_{\text {peak }}\right)$ for positive and negative $3 \sigma$ clumps. Each clump is associated with a colour based on the number of pixels detected in the core. While higher $S N R_{\text {peak }}$ are observed for positive detections, a noise contribution still affects both the positive and negative sides.

pixels $i$ and channels $k$, with the major (resp. minor) axis size $\sigma_{\text {maj }}\left(\right.$ resp. $\left.\sigma_{\text {min }}\right)$ as follows:

$\begin{aligned} \sigma_{\text {maj }}\left(\Phi_{\text {iso }}\right) & =\sqrt{\frac{\sum_{k}^{\text {iso }} \sum_{i}^{\text {iso }} \Phi_{\mathrm{i}, \mathrm{k}}\left[x_{\mathrm{i}}-\bar{x}\left(\Phi_{\text {iso }}\right)\right]^{2}}{\sum_{k}^{\text {iso }} \sum_{i}^{\text {iso }} \Phi_{\mathrm{i}, \mathrm{k}}}} \\ \sigma_{\text {min }}\left(\Phi_{\text {iso }}\right) & =\sqrt{\frac{\sum_{k}^{\text {iso }} \sum_{i}^{\text {iso }} \Phi_{\mathrm{i}, \mathrm{k}}\left[y_{\mathrm{i}}-\bar{y}\left(\Phi_{\mathrm{iso}}\right)\right]^{2}}{\sum_{k}^{\text {iso }} \sum_{i}^{\mathrm{iso}} \Phi_{\mathrm{i}, \mathrm{k}}}}\end{aligned}$

where $\Phi_{\mathrm{i}, \mathrm{k}}$ is the flux in $\mathrm{Jy} \mathrm{beam}^{-1}$ over all channel $k$ and pixel $i$, respectively. The quantities $x_{i}$ and $y_{i}$ refer to the offset positions along the major and minor axis, respectively. The quantities $\bar{x}$ and $\bar{y}$ are the coordinates of barycentre of each isophote in the following:

$\bar{x}\left(\Phi_{\text {iso }}\right)=\frac{\sum_{k}^{\text {iso }} \sum_{i}^{\text {iso }} \Phi_{\mathrm{i}, \mathrm{k}} x_{\mathrm{i}}}{\sum_{k}^{\text {iso }} \sum_{i}^{\text {iso }} \Phi_{\mathrm{i}, \mathrm{k}}}, \bar{y}\left(\Phi_{\text {iso }}\right)=\frac{\sum_{k}^{\text {iso }} \sum_{i}^{\text {iso }} \Phi_{\mathrm{i}, \mathrm{k}} y_{\mathrm{i}}}{\sum_{k}^{\text {iso }} \sum_{i}^{\text {iso }} \Phi_{\mathrm{i}, \mathrm{k}}}$.

Similarly, the velocity dispersion can be defined as

$\sigma_{\mathrm{v}}\left(\Phi_{\text {iso }}\right)=\sqrt{\frac{\sum_{k}^{\text {iso }} \sum_{i}^{\text {iso }} \Phi_{\mathrm{i}, \mathrm{k}}\left[v_{\mathrm{k}}-\bar{v}\left(\Phi_{\text {iso }}\right)\right]^{2}}{\sum_{k}^{\text {iso }} \sum_{i}^{\text {iso }} \Phi_{\mathrm{i}, \mathrm{k}}}}$,

where

$\bar{v}\left(\Phi_{\text {iso }}\right)=\frac{\sum_{k}^{\text {iso }} \sum_{i}^{\text {iso }} \Phi_{\mathrm{i}, \mathrm{k}} v_{\mathrm{k}}}{\sum_{k}^{\text {iso }} \sum_{i}^{\text {iso }} \Phi_{\mathrm{i}, \mathrm{k}}}$

and $v_{\mathrm{k}}$ is the velocity at channel $k$. Last, the total integrated flux in $\mathrm{Jy} \mathrm{km} \mathrm{s}^{-1}$ can be computed as

$F_{\mathrm{CO}}\left(\Phi_{\text {iso }}\right)=\sum_{k}^{\text {iso }} \sum_{i}^{\text {iso }} \Phi_{\mathrm{i}, \mathrm{k}} \delta x \delta y \delta v$, where $\delta x$ and $\delta y$ are the size of the spatial pixels in arcsec and $\delta v$ is the spectral resolution in $\mathrm{km} \mathrm{s}^{-1}$. In order to find the values of the size, velocity dispersion, and flux of the clumps, we use a weighted (by the number of pixels in each isophote) linear least-squares regression to extrapolate the moments at $\Phi_{\text {iso }}=\Phi_{0}=0 \mathrm{Jy}_{\text {beam }}{ }^{-1}$. Figure 3 (resp. Fig. B.3) shows the principle of this procedure applied to the determination of the parameters of the strongest cloud (resp. the cloud analysed in Melchior \& Combes 2017), we obtain the values of $\sigma_{\text {maj }}\left(\Phi_{0}\right)$, $\sigma_{\min }\left(\Phi_{0}\right), \sigma_{\mathrm{v}}\left(\Phi_{0}\right)$, and $F_{\mathrm{CO}}\left(\Phi_{0}\right)$. For comparison purposes, we also include values of the velocity dispersion and flux measured through Gaussian fit within the GILDAS environment. We observe a good agreement between the two methods. In order to get consistent measurements we subsequently use the extrapolated $\sigma_{\mathrm{v}}$. Gratier et al. (2012) and Corbelli et al. (2017) adopted the Class/Gildas measurements for the velocity dispersions and integrated fluxes as they provide lower uncertainties. As further explained below, we compute errors on the extrapolated values the same way as Rosolowsky \& Leroy (2006) with the bootstrap method, and we find uncertainties compatible with the Class measurements based on a Gaussian fit on the integrated spectra.

We find the uncertainty over the extrapolated parameters via the bootstrapping method, which is a robust technique to estimate the error when it is difficult to determine formally the contribution of each source of noise. We proceed as follows: each isophote contains a number $\mathrm{N}$ of pixels, we choose randomly $\mathrm{N}$ pixels with replacement, and so on for each isophote. A new extrapolation is made from this bootstrapped clump. This process is repeated 500 times and the standard deviation of the distribution of extrapolated values multiplied by the square root of the number of pixels in a beam, i.e. the oversampling rate (to account for pixels being correlated), is used as the uncertainty. The resulting uncertainties can be seen in Fig. 3 and in Table 3, which lists our final sample.

The rms spatial size $\sigma_{\mathrm{r}}$ of each clump is then calculated by deconvolving the spatial beam from second moments of the extrapolated clump size, assuming the clumps have the shape of a $2 \mathrm{D}$ Gaussian, and substracting the RMS beam sizes $\sigma_{\text {beam,maj }}$ and $\sigma_{\text {beam,min }}$ as follows:

$\sigma_{\mathrm{r}}=\left(\sqrt{\sigma_{\text {maj }}^{2}\left(\Phi_{0}\right)-\sigma_{\text {beam,maj }}^{2}} \sqrt{\sigma_{\text {min }}^{2}\left(\Phi_{0}\right)-\sigma_{\text {beam,min }}^{2}}\right)^{1 / 2}$,

where $\sigma_{\text {maj }}\left(\Phi_{0}\right)>\sigma_{\text {beam,maj }}$ and $\sigma_{\text {min }}\left(\Phi_{0}\right)>\sigma_{\text {beam,min }}$, a condition met for all our sample, including negative clumps. We compare the moments of major and minor size extrapolated at $\Phi_{0}$ to the RMS size of the beam, not to the projection at $\Phi_{0}$ of the beam size. This is a robust approach to take into account the resolution bias when clumps are barely resolved. This method shows high performances on marginal resolution, low $\mathrm{S} / \mathrm{N}$ molecular clouds (Rosolowsky \& Leroy 2006). While the beam has a position angle of $70^{\circ}$, the mean inclination of the major axis of our 

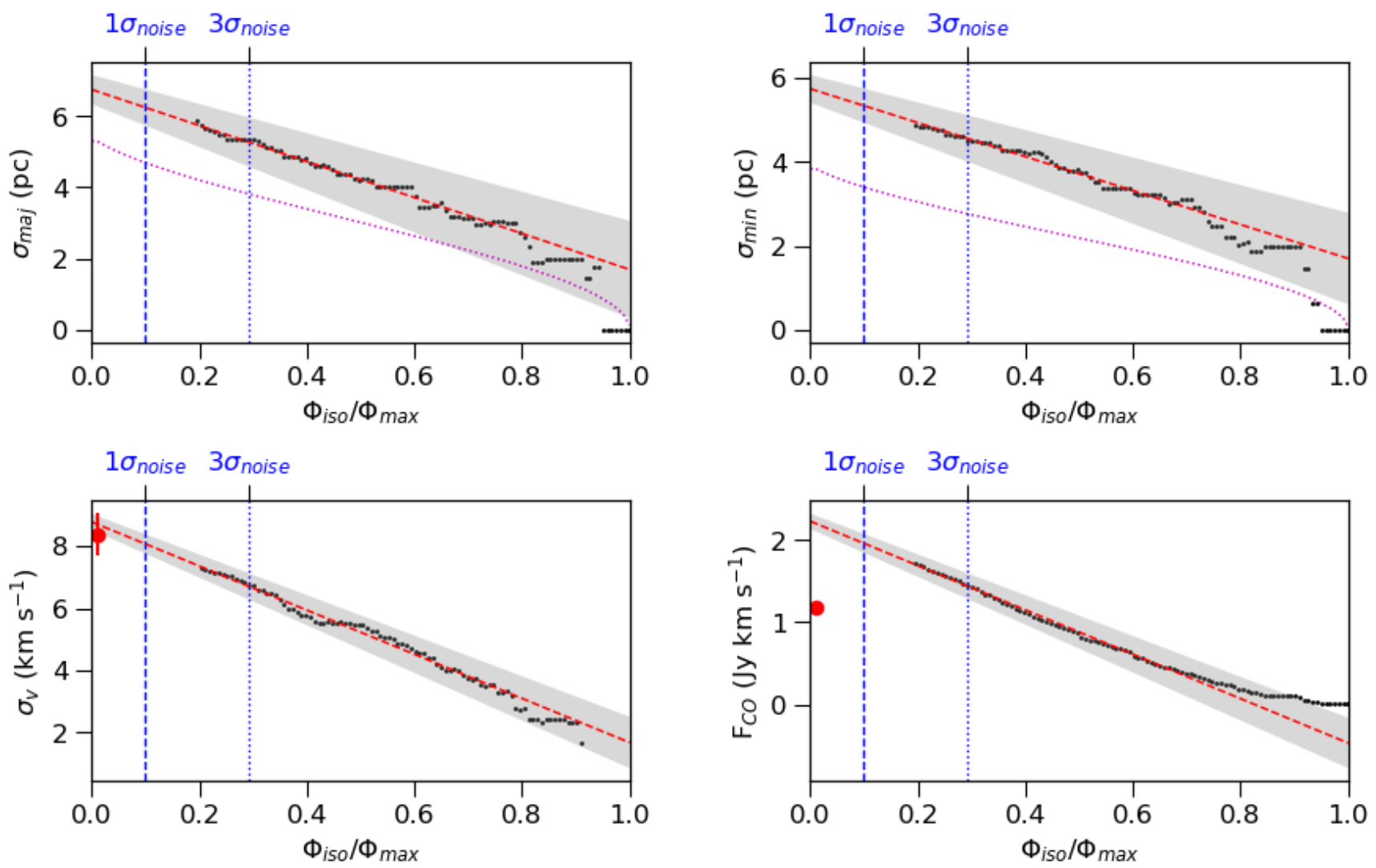

Fig. 3. Extrapolation method applied on the highest $\mathrm{S} / \mathrm{N}$ clump at $\mathrm{J} 2000$ coordinates $00^{\mathrm{h}} 42^{\mathrm{m}} 43.9^{\mathrm{s}}$ and $+41^{\circ} 15^{\prime} 35.84^{\prime \prime}$ with $S N R^{\text {peak }}=10.3$ and $S N R^{\text {tot }}=64.4$. We perform extrapolations to the 0-level with a linear regression (red dashed line) on the data for spatial size $\sigma_{\text {maj }}$ and $\sigma_{\text {min }}$ (top panels), velocity dispersion $\sigma_{\mathrm{v}}$ distribution (bottom left panel), and total flux $F_{\mathrm{CO}}$ (bottom right panel). The uncertainty is computed with

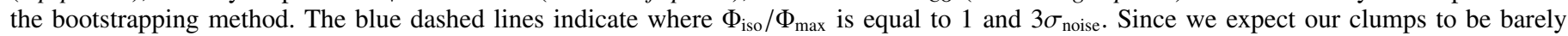
resolved, we show as a magenta dashed line the size for a perfect Gaussian PSF, noiseless, and simulated equivalent case. The red dots indicate the values found by fitting a Gaussian for the velocity distribution and total flux. The size over the major and minor axis is slightly larger than that obtained for the simulated clump, which suggests that this clump is barely resolved. The velocity dispersion is about 4 times the spectral resolution $\left(2.2 \mathrm{~km} \mathrm{~s}^{-1}\right)$. There is a good agreement between the extrapolated velocity dispersions and those directly measured on the spectra, while the total flux is slightly higher for the extrapolation.

clumps is $(82 \pm 25)^{\circ}$, indicating that some clumps are resolved and exhibit structures and most structures are unresolved. This influences the $\sigma_{\mathrm{r}}$ calculation, hence we assume the clumps to be spherical which might lead to a slight underestimation of the spatial size.

The equivalent radius $R_{\text {eq }}$ of the assumed spherical clump is chosen following the empirical definition from Solomon et al. (1987), that is $R_{\mathrm{eq}} \approx 1.91 \sigma_{\mathrm{r}}$. Rosolowsky \& Leroy (2006) estimate that if clumps behave as spherical clouds with a density profile $\rho \propto r^{-\beta}, \sigma_{\mathrm{r}}^{2}(\beta)=\frac{3-\beta}{3(5-\beta)} R^{2}$ and $R(\beta=1)=\sqrt{6} \sigma_{\mathrm{r}} \sim 2.45 \sigma_{\mathrm{r}}$. This value is larger than $1.91 \sigma_{\mathrm{r}}$ and can be explained by the behaviour of the ${ }^{12} \mathrm{CO}$, which shows a shallower density profile than the used model. As this $R_{\text {eq }}$ radius is also used in Rosolowsky \& Leroy (2006), it provides an adequate approximation that permits comparison. From now on, we refer to $\sigma_{\text {maj }}\left(\Phi_{0}\right)$, $\sigma_{\text {min }}\left(\Phi_{0}\right), \sigma_{\mathrm{v}}\left(\Phi_{0}\right)$, and $F_{\mathrm{CO}}\left(\Phi_{0}\right)$, as $\sigma_{\text {maj }}, \sigma_{\text {min }}, \sigma_{\mathrm{v}}$, and $F_{\mathrm{CO}}$ since only the extrapolated values hold any relevance in this work.

From these properties, we can compute the $\mathrm{S} / \mathrm{N} S N R_{\text {tot }}$ based on the total flux $F_{\mathrm{CO}}$ and the noise of the spectrum integrated within the box containing each clump.

\subsection{Velocity coherence and side lobes}

It is unlikely that two genuine clumps would have identical velocities, and we expect a velocity gradient up to $600 \mathrm{~km} \mathrm{~s}^{-1}$ in this region. Hence, it is highly probable that two clumps with the exact same velocity but different intensities correspond to one genuine clump and a side lobe which should be rejected from our selection. As seen in Fig. B.1, which shows the maps of positive and negative signals and the corresponding velocity distributions, a noticeable amount of clumps display similar or identical velocities. The cleaning of side lobes is done in the GILDAs environment. A CLEAN algorithm (Gueth et al. 1995) is run over each channel where more than one clump is detected and a support is centred on the strongest clump. To assess the robustness of signals, we keep negative clumps and side lobes in the first step to explore their behaviour statistically, as described below.

\subsection{Principal component analysis}

We applied PCA to the full sample of molecular cloud candidates (both negative and positive clumps), using the following parameters: $S N R_{\text {peak }}, S N R_{\text {tot }}, R_{\text {eq }}$, and $\sigma_{\mathrm{v}}$. These four parameters are first standardised as follows: $\widetilde{T}=(T-\langle T\rangle) / \sigma_{\mathrm{T}}$, where $\mathrm{T}$ is the studied parameter, $\langle T\rangle$ its mean value, and $\sigma_{\mathrm{T}}$ its standard deviation. Second, with the Python ScikitLearn library, we extracted two principal components, corresponding to the eigenvectors with the highest eigenvalues. These are a linear combination of the four standardised parameters and are written as 

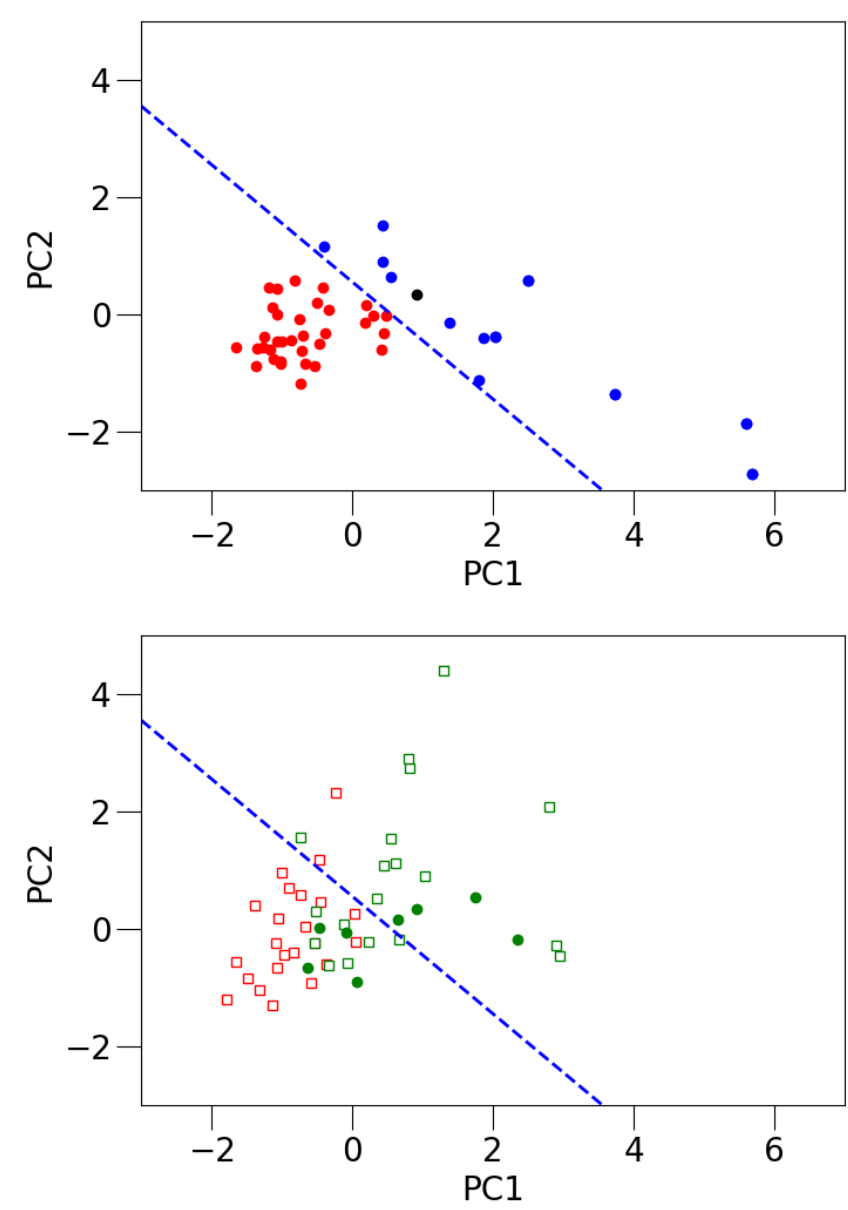

Fig. 4. Results of the PCA analysis. The principal components PC1 and $\mathrm{PC} 2$ are defined in Eq. (8). Clumps with positive (resp. negative) signals are shown in full circles (resp. empty squares). Top panel: map from the PCA for positive signals. Selected (resp. rejected) clumps are shown in blue (resp. red). The blue dashed straight line shows the limit between selected and rejected clumps and follows the relation $P C 2=$ $-1 \times P C 1+0.55$. Bottom panel: map for rejected signals: negative signals and side lobes. Side lobes, identified in Sect. 2.4, are shown in green, and the other negative signals not identified as side lobes are indicated in red. We note that side lobes can have a high S/N. Most side lobes correspond to negative signals, but we also eliminate four side lobes with a positive signal.

$$
\begin{aligned}
& P C 1=0.23 \widetilde{R_{\text {eq }}}+0.57 S \widetilde{S / N_{\text {peak }}}+0.67 \overparen{S / N_{\text {tot }}}+0.41 \widetilde{\sigma_{\mathrm{v}}} \\
& P C 2=0.92 \widetilde{R_{\text {eq }}}-0.38 S \widetilde{S N_{\text {peak }}}+0.01 \widetilde{S / N_{\text {tot }}}+0.001 \widetilde{\sigma_{\mathrm{v}}}
\end{aligned}
$$

Figure 4 maps the sample on two axes defined as the two main principal components accounting for $50 \%$ and $25 \%$ of the behaviour of the sample. The top panel maps the clumps kept by the cleaning procedure, while the bottom panel represents the identified side lobes and negative clumps. After visual inspection and cross-check with the negative signals, we eliminated the red dots in the top panel of Fig. 4 below the blue dashed line. Hence, on the top panel, we kept blue dots with

$$
1.15 \widetilde{R_{\mathrm{eq}}}+0.68 \widetilde{S / N_{\text {tot }}}+0.41 \widetilde{\sigma_{\mathrm{v}}}+0.19 S \widetilde{S / N_{\text {peak }}} \geq 0.55 \text {. }
$$

The bottom panel shows candidates rejected as side lobes and/or exhibiting a negative signal. This shows the limitation of this analysis. Amplitude of side lobes can be important and four positive side lobes with relatively high significance with respect to the selected signals (in the top panel) are rejected.
We thus kept 12 clumps whose parameters are shown in Table 3. They have $S N R_{\text {peak }} \geq 4.2$ and $S N R_{\text {tot }} \geq 15.2$ as indicated in Fig. 5. The maps and corresponding spectra are shown in Fig. 6.

\subsection{Final sample selected}

We show the maps and integrated spectrum for all 12 selected clumps in Fig. 6, by order of decreasing $S N R_{\text {tot }}$. As said in Sect. 2.3, the position angle of several clumps differs significantly from the position angle of the beam. It tends to happen for extended clumps that have structures.

We selected the sample via a five-step procedure:

1. In Sect. 2.2, the $3 \sigma$ peak detection allowed us to find 54 positive candidates within the data cube, including faint signals which needed further investigation. At this stage, 47 negative clumps were kept in order to perform a statistical analysis: those negative (false) detection with similar characteristics compared to our sample of positive candidates provide statistical information on the noise affecting the data. The $S N R_{\text {peak }}$ of each core was calculated.

2. In Sect. 2.3, we extrapolated the values of $\sigma_{\text {maj }}, \sigma_{\text {min }}, \sigma_{\mathrm{v}}$, and $F_{\mathrm{CO}}$ for both positive and negative clumps. This allowed us to find the values for $R_{\mathrm{eq}}$ and $S N R_{\mathrm{tot}}$.

3. In Sect. 2.4, with the use of CLEAN algorithm, we checked clumps with identical velocities for side lobes. Positive and negative clumps, some with a significant $S N R_{\text {tot }}$ were found to be side lobes of three positive $3 \sigma$ clumps.

4. In Sect 2.5, we applied a statistical analysis on our sample of clumps.

Hence, we complete the selection procedure. The final map is presented in Fig. 7, with the clump mean velocity in colour. Although the $\mathrm{CO}$ emission is patchy, it follows the general velocity field found in $\mathrm{H} \alpha$ (Boulesteix et al. 1987). The $\mathrm{CO}$ emission globally corresponds well to the star formation map of Ford et al. (2013). We kept 12 clumps with $S N R_{\text {tot }}$ showed in Fig. 5. Their properties are summed up in Table 3. The distribution of $\sigma_{\text {maj }}$, $\sigma_{\text {min }}, \sigma_{\mathrm{v}}$, and $F_{\mathrm{CO}}$ for selected clumps is shown in Fig. 8.

\section{Cloud properties}

In this section, our goal is to determine the physical properties of the identified molecular clouds in the centre of M31, size, velocity dispersion, mass, and virial ratio, to compare these to nearby galaxy clouds and to their scaling relations.

\subsection{Velocity dispersion}

The average velocity dispersion of our selected sample, computed as in Eq. (4), is $6.8 \pm 1.3 \mathrm{~km} \mathrm{~s}^{-1}$. The corresponding histogram is shown in Fig. 8. Caldù-Primo \& Schruba (2016) detected molecular clouds, mostly located in the $10 \mathrm{kpc}$ ring of M 31, found a median velocity dispersion of $3.1 \pm 1.1 \mathrm{~km} \mathrm{~s}^{-1}$ with interferometric data.

We find a median velocity dispersion of $6.35 \mathrm{~km} \mathrm{~s}^{-1}$. However, the relation velocity dispersion and radius do not follow the expected Larson's relation (cf. Fig. 10), contrary to the massradius relation, as discussed below. While averaging over the whole extent of the clouds, it is possible to find a dispersion as low as $3 \mathrm{~km} \mathrm{~s}^{-1}$ in one clump; the corresponding full width at half maximum (FWHM) would be $\sim 7 \mathrm{~km} \mathrm{~s}^{-1}$. The present spectral resolution, and sampling (FWHM of $5.07 \mathrm{~km} \mathrm{~s}^{-1}$ ), is a strong limitation in our determinations. 
Table 3. Properties of the 12 selected molecular clumps extracted from our data cube.

\begin{tabular}{|c|c|c|c|c|c|c|c|c|c|c|}
\hline \multirow[b]{2}{*}{ ID } & \multirow[b]{2}{*}{$\begin{array}{l}\text { Offsets } \\
(\operatorname{arcsec})\end{array}$} & \multirow[b]{2}{*}{$\begin{array}{c}V_{0} \\
\left(\mathrm{~km} \mathrm{~s}^{-1}\right)\end{array}$} & \multirow[b]{2}{*}{$\begin{array}{c}\Phi_{\max } \\
\left(\mathrm{mJy}_{\text {beam }}^{-1}\right)\end{array}$} & \multirow[b]{2}{*}{$\begin{array}{c}\sigma_{\text {noise }} \\
\left(\mathrm{mJy} \mathrm{beam}^{-1}\right)\end{array}$} & \multirow[b]{2}{*}{$S N R_{\text {peak }}$} & \multicolumn{5}{|c|}{ Extrapolations } \\
\hline & & & & & & $\begin{array}{l}\sigma_{\text {maj }} \\
(\mathrm{pc})\end{array}$ & $\begin{array}{l}\sigma_{\min } \\
(\mathrm{pc})\end{array}$ & $\begin{array}{c}\sigma_{\mathrm{v}} \\
\left(\mathrm{km} \mathrm{s}^{-1}\right)\end{array}$ & $\begin{array}{c}F_{\mathrm{CO}} \\
\left(\mathrm{Jy} \mathrm{km} \mathrm{s}^{-1}\right)\end{array}$ & $S N R_{\mathrm{tot}}$ \\
\hline 1 & $-5.2,-32.5$ & $45.1 \pm 0.7$ & 55.8 & 5.4 & 10.3 & $6.6 \pm 0.4$ & $5.7 \pm 0.3$ & $8.8 \pm 0.3$ & $2.23 \pm 0.09$ & 64.4 \\
\hline 2 & $30.8,2.3$ & $145.5 \pm 0.4$ & 58.4 & 4.5 & 12.9 & $6.0 \pm 0.4$ & $5.8 \pm 0.4$ & $6.0 \pm 0.3$ & $1.48 \pm 0.09$ & 56.9 \\
\hline 3 & $34.4,-3.8$ & $110.9 \pm 1.5$ & 26.8 & 5.6 & 4.8 & $9.7 \pm 0.5$ & $7.6 \pm 0.4$ & $7.8 \pm 0.2$ & $1.42 \pm 0.05$ & 49.4 \\
\hline 4 & $29.0,10.8$ & $76.6 \pm 0.7$ & 34.8 & 3.7 & 9.4 & $8.1 \pm 0.4$ & $5.9 \pm 0.3$ & $6.0 \pm 0.2$ & $1.10 \pm 0.06$ & 46.4 \\
\hline 5 & $13.1,-22.7$ & $112.9 \pm 1.2$ & 29.9 & 5.1 & 5.9 & $8.7 \pm 0.5$ & $5.5 \pm 0.3$ & $6.7 \pm 0.2$ & $1.15 \pm 0.05$ & 39.6 \\
\hline 6 & $0.9,-45.9$ & $-35.8 \pm 0.9$ & 48.1 & 7.7 & 6.3 & $5.4 \pm 0.3$ & $5.4 \pm 0.3$ & $6.5 \pm 0.2$ & $1.98 \pm 0.07$ & 36.0 \\
\hline 7 & $19.8,5.9$ & $65.8 \pm 1.4$ & 20.5 & 4.1 & 5.0 & $9.2 \pm 0.7$ & $4.3 \pm 0.3$ & $10.7 \pm 0.3$ & $0.73 \pm 0.03$ & 31.1 \\
\hline 8 & $35.7,0.4$ & $171.5 \pm 1.5$ & 27.7 & 5.0 & 5.5 & $7.3 \pm 0.4$ & $7.3 \pm 0.4$ & $8.7 \pm 0.3$ & $0.75 \pm 0.04$ & 23.8 \\
\hline 9 & $2.1,-9.9$ & $4.3 \pm 1.5$ & 15.5 & 3.2 & 4.8 & $11.4 \pm 0.5$ & $7.9 \pm 0.3$ & $4.4 \pm 0.2$ & $0.44 \pm 0.02$ & 22.8 \\
\hline 10 & $7.6,-24.0$ & $95.8 \pm 1.0$ & 21.4 & 5.1 & 4.2 & $10.7 \pm 0.6$ & $10.6 \pm 0.5$ & $6.2 \pm 0.2$ & $0.65 \pm 0.03$ & 19.0 \\
\hline 11 & $-22.3,-33.7$ & $-167.6 \pm 1.4$ & 24.0 & 5.7 & 4.2 & $9.9 \pm 0.4$ & $9.5 \pm 0.3$ & $3.4 \pm 0.2$ & $0.57 \pm 0.03$ & 15.4 \\
\hline 12 & $1.5,1.7$ & $-176.5 \pm 1.1$ & 19.1 & 3.5 & 5.4 & $10.8 \pm 0.7$ & $7.8 \pm 0.5$ & $6.1 \pm 0.2$ & $0.39 \pm 0.02$ & 15.2 \\
\hline
\end{tabular}

Notes. The offsets are calculated according to the optical centre of M 31 at coordinates at J2000 coordinates $00^{\mathrm{h}} 42^{\mathrm{m}} 44.37^{\mathrm{s}}$ and $+41^{\circ} 16^{\prime} 8.34^{\prime \prime}$ (Crane et al. 1992). The value $V_{0}$ is the velocity at the peak flux $\Phi_{\max }$ and the noise of the associated spectrum is $\sigma_{\text {noise. }}$ The $S N R_{\text {peak }}$ is derived from these.The values $\sigma_{\mathrm{v}}, R_{\mathrm{eq}}$, and $F_{\mathrm{CO}}$ are the extrapolations found through the methodology in Sect. 2.3. The $S N R_{\mathrm{tot}}$ is found with $F_{\mathrm{CO}}$ and the noise of the associated integrated spectrum.
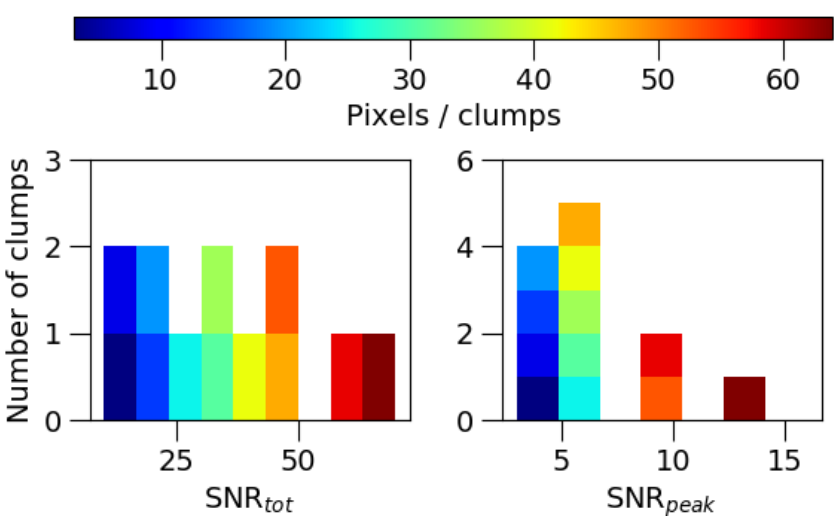

Fig. 5. Signal-to-noise ratio distribution of the selected clumps. The distribution of $S N R_{\text {tot }}$ shows a clear correlation between cores with large number of pixels and high $\mathrm{S} / \mathrm{N}$.

\subsection{Mass estimates}

The luminous mass $M_{\mathrm{H}_{2}}$ of the clumps can be derived from the total integrated flux. First we calculate the CO luminosity $L_{\mathrm{CO}}^{\prime}$, which is defined as (Solomon et al. 1997)

$$
\frac{L_{\mathrm{CO}}^{\prime}}{\mathrm{K} \mathrm{km} \mathrm{s}^{-1} \mathrm{pc}^{2}}=\frac{3.25 \times 10^{7}}{(1+z)^{3}} \frac{F_{\mathrm{CO}}}{\mathrm{Jy} \mathrm{km} \mathrm{s}^{-1}}\left(\frac{D_{\mathrm{L}}}{\mathrm{Mpc}}\right)^{2}\left(\frac{v_{\text {rest }}}{\mathrm{GHz}}\right)^{-2} \text {, }
$$

where $D_{\mathrm{L}}=780 \mathrm{kpc}$ is the distance to $\mathrm{M} 31, v_{\text {rest }}=115.271 \mathrm{GHz}$ is the observed frequency, and $z=0$. This leads to the luminous mass

$\frac{M_{\mathrm{H}_{2}}}{M_{\odot}}=4.4 \frac{L_{\mathrm{CO}}^{\prime}}{\mathrm{Kkm} \mathrm{s}^{-1} \mathrm{pc}^{2}} \frac{X_{\mathrm{CO}}}{2 \times 10^{20} \mathrm{~cm}^{-2}\left(\mathrm{~K} \mathrm{~km} \mathrm{~s}^{-1}\right)^{-1}}$,

where $\mathrm{X}_{\mathrm{CO}}$ is the $\mathrm{CO}$-to- $\mathrm{H}_{2}$ conversion factor (Bolatto et al. 2013). The mass is then given by $M_{\mathrm{H}_{2}}=\alpha_{\mathrm{CO}} L_{\mathrm{CO}}^{\prime}$, where $\alpha_{\mathrm{CO}}=$ $4.36 M_{\odot}\left(\mathrm{K} \mathrm{km} \mathrm{s}^{-1} \mathrm{pc}^{2}\right)^{-1}$, based on the conversion factor of the Milky Way $3.2 M_{\odot}\left(\mathrm{K} \mathrm{km} \mathrm{s}^{-1} \mathrm{pc}^{2}\right)^{-1}$ corrected by a factor 1.36 to account for interstellar helium (Tacconi et al. 2010). We find a total $\mathrm{H}_{2}$ mass of $(8.4 \pm 0.4) \times 10^{4} M_{\odot}$, which is coherent with Melchior \& Combes (2013) estimation of a minimum total mass of $4.2 \times 10^{4} M_{\odot}$. We have a mean $M_{\mathrm{H}_{2}}$ of $(7.0 \pm 0.3) \times 10^{3} M_{\odot}$ for the detected clouds. We find our values for the mass and size of clumps to be consistent with Larson's mass - size relation found in Salomé et al. (2017), Gratier et al. (2012), Rosolowsky (2007); cf. Fig. 9. It is not the case for the velocity - size and velocity - mass relations though. This might be explained in part by the fact that our minimal value of $\sigma_{\mathrm{v}}$ is biased high by our spectral resolution and selection process; we ask for a minimum of two consecutive channels. Hence, our velocity dispersions are somewhat over-estimated. Although, for signals with $S N R_{\text {peak }}<10$, Rosolowsky \& Leroy (2006) has shown that measurements based on interferometric data tend to underestimate size and velocity dispersion.

The surface density $\Sigma_{\mathrm{H}_{2}}$ is found by dividing the molecular mass of the clump by its area in squared parsecs. The area is calculated for an elliptic shape with the major and minor axis deconvolved from the beam. We find an average surface density of $15.3 \pm 2.6 M_{\odot} \mathrm{pc}^{2}$. This is three times less than in NGC 5128, about two times less than in the Milky Way, but close to the value found in the LMC of $21 \pm 9 M_{\odot} \mathrm{pc}^{2}$ (Salomé et al. 2017; Miville-Deschênes et al. 2017; Hughes et al. 2013).

The molecular column density is calculated as follows:

$N_{\mathrm{H}_{2}}=\frac{\Sigma_{\mathrm{H}_{2}}}{M_{\odot} \mathrm{pc}^{2}} \frac{M_{\odot}}{\mathrm{kg}}\left(\frac{2.3 m_{\mathrm{p}}}{\mathrm{kg}}\right)^{-1}\left(\frac{\mathrm{pc}}{\mathrm{cm}}\right)^{-2}$,

where we multiply the proton mass $m_{\mathrm{p}}$ by 2.3 to take into account the helium mass. The average column density is $(8.5 \pm 1.5) \times$ $10^{20} \mathrm{~cm}^{-2}$

In order to estimate the impact of the magnetic, kinetic, and gravitational energy of the clumps, we finally calculate the virial parameter (Bertoldi \& McKee 1992; McKee \& Zweibel 1992) as

$\alpha_{\mathrm{vir}}=5 \sigma_{\mathrm{v}}^{2} \frac{R_{\mathrm{eq}}}{G M_{\mathrm{H}_{2}}}$.

A value of $\alpha_{\mathrm{vir}} \leq 1$ indicates a gravitationally bound core with a possible support of magnetic fields while if $\alpha_{\text {vir }} \leq 2$, it means the 

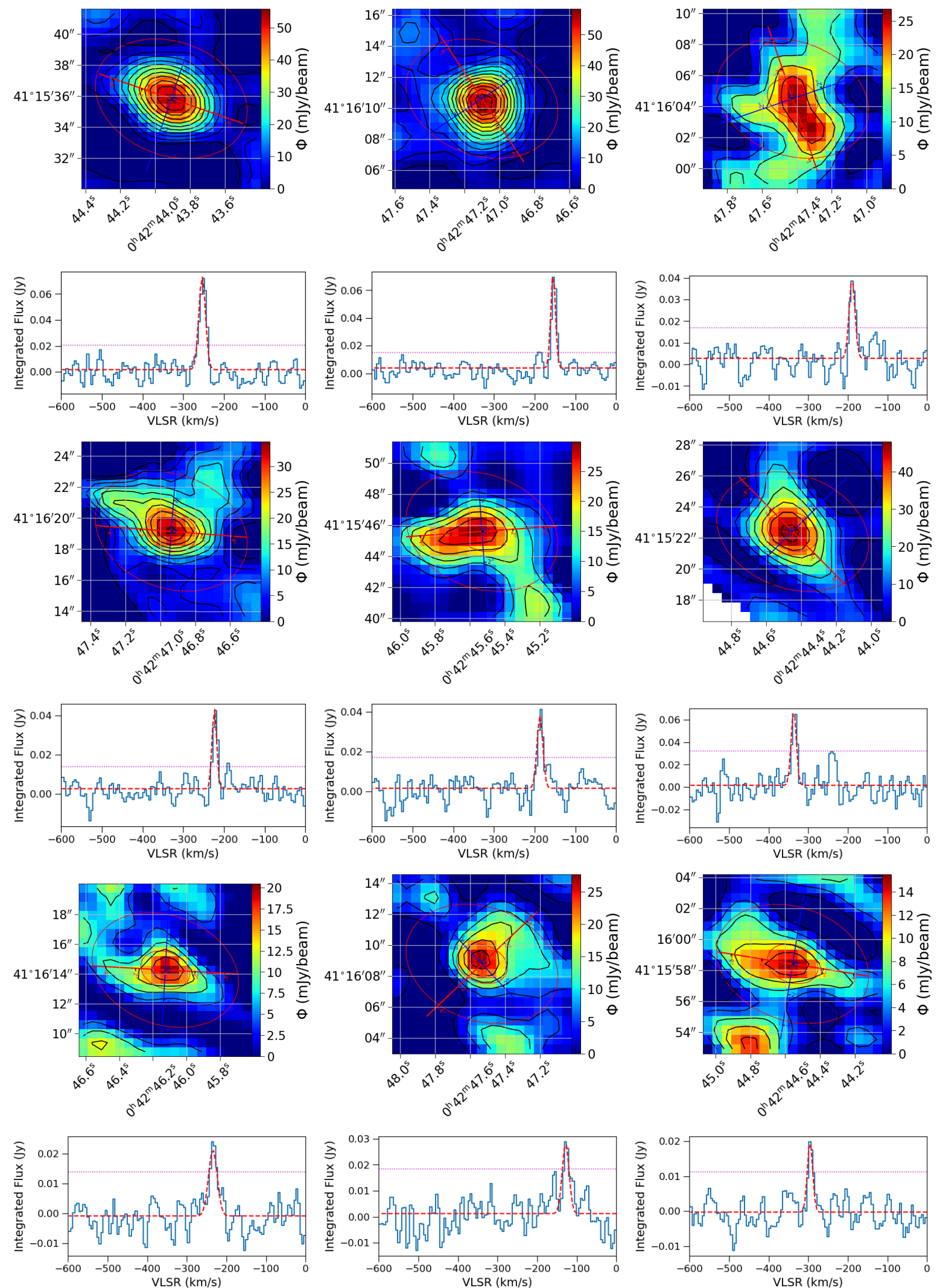

Fig. 6. Maps for the 12 clumps centred on the pixel and channel with peak flux and their associated integrated spectrum, organised by order of decreasing $S N R_{\text {tot }}$ (i.e. ID1 to ID12). The major and minor axes are shown with a red and blue line, respectively. Two ellipses show the area of the FWHM beam and of the box used for the calculation of extrapolation (with major and minor axis three times as big as the beam). The contours are separated by one sigma each; the negative sigma is shown with dashed contours. The spectra are represented in blue. The magenta dashed line in the spectrum is the $3 \sigma$ level used for the selection procedure and the dashed red line corresponds to the Gaussian fit performed over the peak emission. 

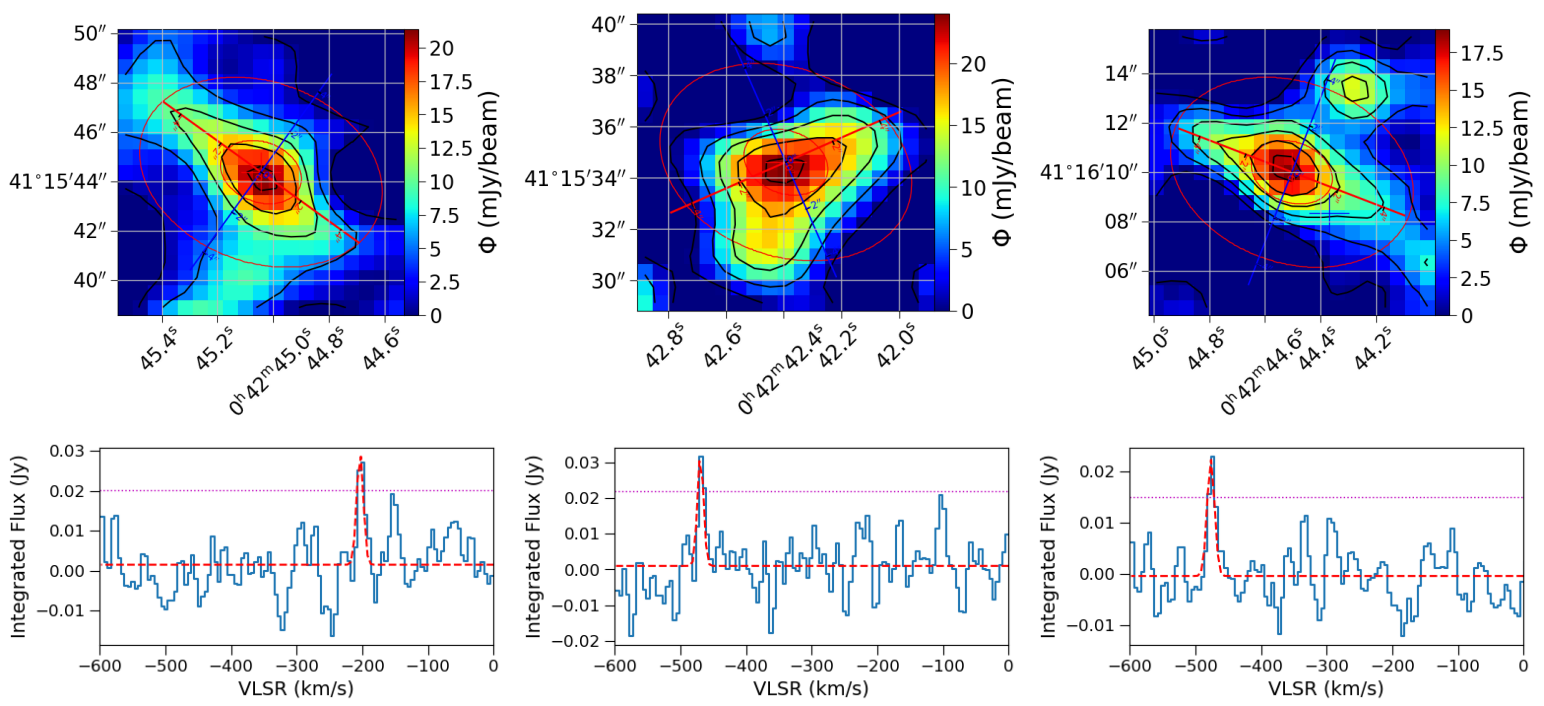

Fig. 6. continued.
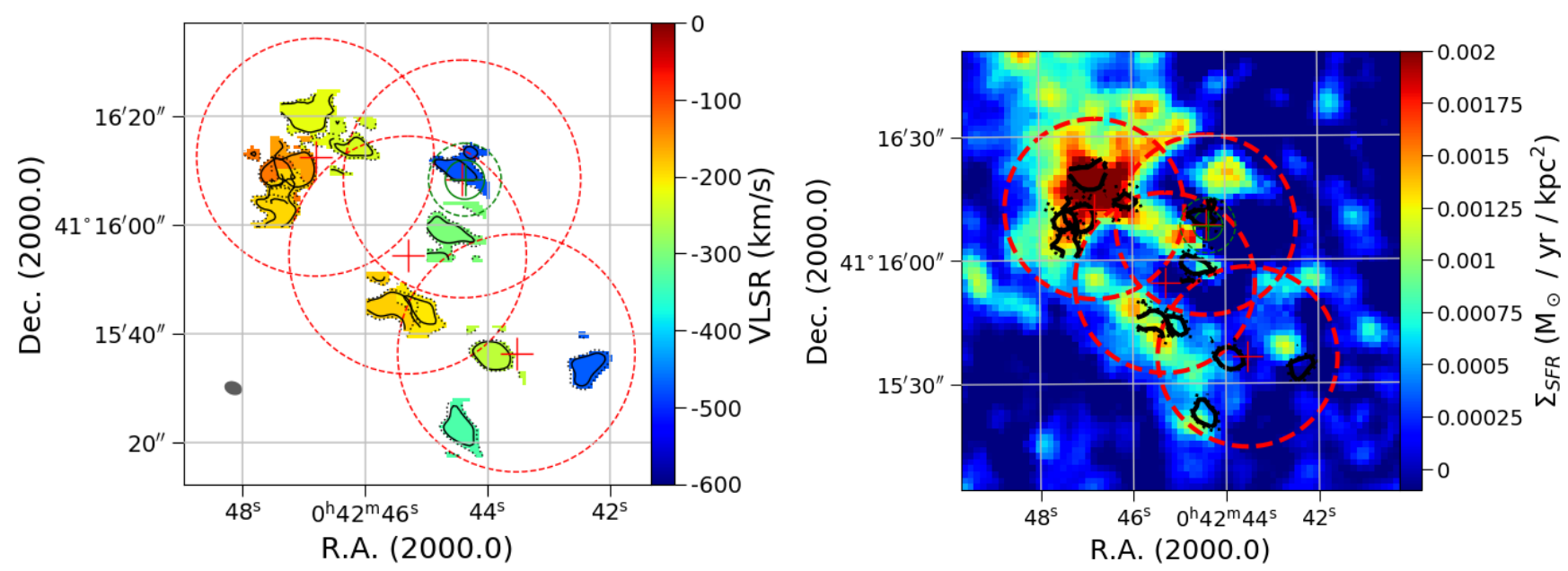

Fig. 7. Left: mean velocity map of the selected clumps with $1 \sigma$ (dotted) and $2 \sigma$ (solid) intensity contours around clump. Right: map of the surface density SFR in M 31 central region from Ford et al. (2013) with the contours of our clumps superposed.

core is gravitationally bound without magnetic support. In our case, clumps have $\alpha_{\text {vir }} \gg 2$, their kinetic energy appears dominant and they are not virialised. Compared to the virial parameter in Salomé et al. (2017), Miville-Deschênes et al. (2017), and Hughes et al. (2013) for giant molecular clouds, our mean value is $140 \pm 72$, which is one to two orders of magnitude higher than the formes values since our clumps are smaller and less massive.

Figure 10 reveals that the velocity dispersions of our measured clumps lie well above the relation for Milky Way clouds. Certainly our spectral resolution prevents us from detecting velocity dispersions below the relation. However, even after deconvolution from the spectral resolution, the majority of clumps have a broad dispersion. We conclude that the virial factors $\alpha_{\text {vir }}$ are in a large majority much larger than 1 and the clumps are not virialised. Certainly, they are transient agglomerations of smaller entities, which might be virialised.

\section{Discussion}

In the following, we discuss how this new molecular gas detection compares with other measurements. In Sect. 4.1, we discuss the line ratios of some clumps based on $\mathrm{CO}(2-1)$ detection from
Melchior \& Combes (2013) and CO(3-2) from Li et al. (2019). In Sect. 4.2, we study the spectral energy distribution of this region and discuss the star formation tracers available in this region. We show the absence of correlation between dust, most probably heated by the interstellar radiation field, and the farultraviolet (FUV) due to the old bulge stellar population plus the $200 \mathrm{Myr}$ inner disc discussed by Lauer et al. (2012). Last, relying on the SFR estimate of this region, in Sect. 4.3 we discuss the extreme position of our measurement on the Kennicutt-Schmidt law.

\section{1. $C O(2-1)$-to- $C O(1-0)$ line ratio}

\subsubsection{Data}

In Melchior \& Combes (2013), we analysed IRAM 30 m CO(21) data obtained with the HERA instrument. These data overlap the IRAM-PdB CO(1-0) mosaic studied in this work. We do not find a priori a one-to-one correspondence with the signals detected in Melchior \& Combes (2013). The $\mathrm{CO}(2-1)$ signal is detected in more regions than $\mathrm{CO}(1-0)$. However, many detections were below $4 \sigma$ and were not recovered by the IRAM-PdB 

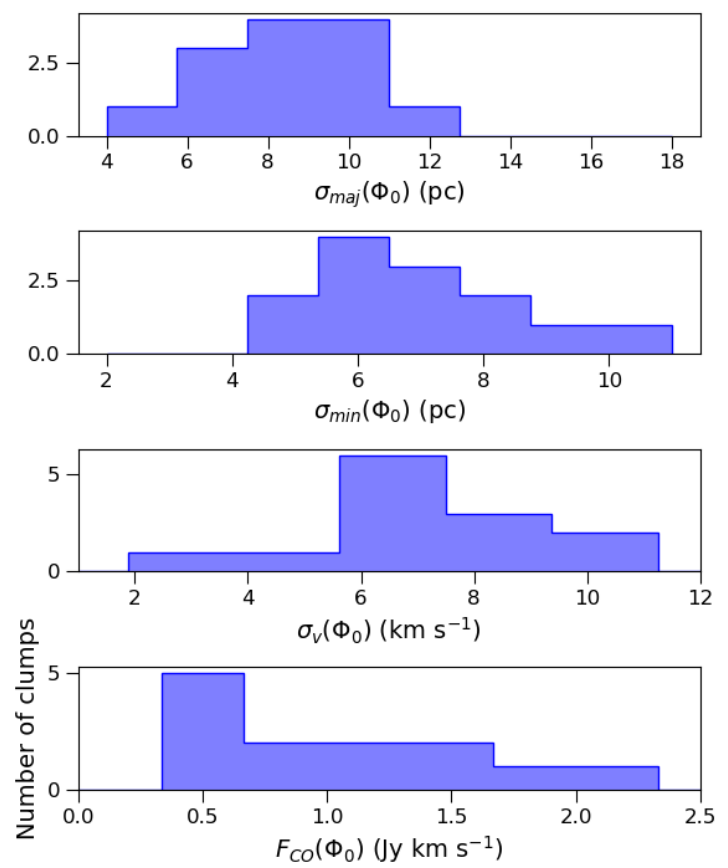

Fig. 8. Distribution of the $\sigma_{\text {maj }}, \sigma_{\text {min }}, \sigma_{\mathrm{v}}$, and $F_{\mathrm{CO}}$ for the selected clumps. We find the average values $\left\langle\sigma_{\text {maj }}\right\rangle=8.6 \pm 2.5 \mathrm{pc},\left\langle\sigma_{\min }\right\rangle=$ $7.0 \pm 1.7 \mathrm{pc},\left\langle\sigma_{\mathrm{v}}\right\rangle=6.8 \pm 1.1 \mathrm{~km} \mathrm{~s}^{-1}$, and $\left\langle F_{\mathrm{CO}}\right\rangle=1.1 \pm 0.6 \mathrm{Jy} \mathrm{km} \mathrm{s}^{-1}$.

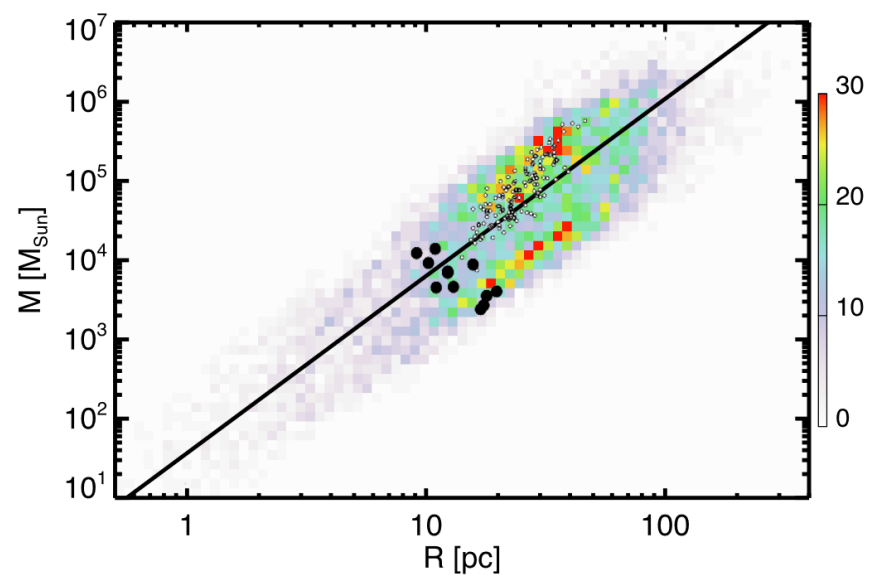

Fig. 9. Mass-size relation for the M31 circum-nuclear clumps (large full black symbols) superposed on the relation obtained for Milky Way clouds by Miville-Deschênes et al. (2017). The colour scale is proportional to the density of points. The solid line indicates a linear fit of slope 2.2. The small empty symbols denote the clouds identified in Centaurus A northern filament by Salomé et al. (2017).

data presented here. While this is not surprising given the low $\mathrm{S} / \mathrm{N}$, the signal could be affected by interferometric filtering if the gas is extended. Indeed, Rosolowsky \& Leroy (2006) found that for a given mass a significant fraction of the flux can be lost when the peak $\mathrm{S} / \mathrm{N}$ is lower than 10.3 spectra (labelled 15,28 , and 36 in Melchior \& Combes 2013) which are actually detected above $4 \sigma$ in $\mathrm{CO}(2-1)$ and have counterparts in the $\mathrm{CO}(1-0)$ interferometric data cube. Each $\mathrm{CO}(2-1)$ pointing corresponds to a 12 arcsec HPBW. These $\mathrm{CO}(1-0)$ maps of these three simultaneous $\mathrm{CO}(1-0)$ and $\mathrm{CO}(2-1)$ detections are shown in Fig. 11 and correspond to the clumps $\mathrm{N}^{o} 1,2$, and 5 in Table 3 . As shown in this figure, the $\mathrm{CO}(2-1)$ detections are not centred on the $\mathrm{CO}(1-0)$ peak of emission and the $\mathrm{CO}(1-0)$ clumps do not fill

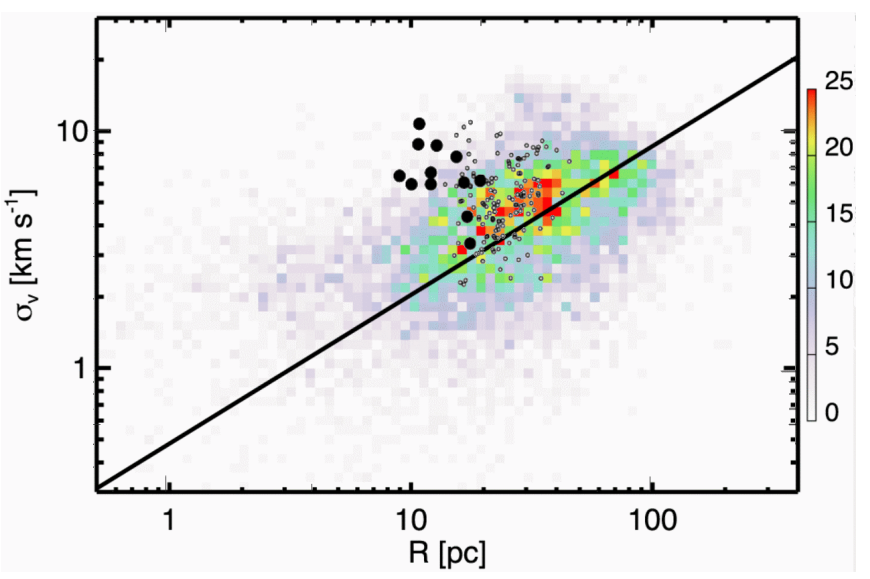

Fig. 10. Velocity dispersion-size relation for the M31 circum-nuclear clumps (large full black symbols) superposed on the relation obtained for Milky Way clouds by Miville-Deschênes et al. (2017). Like in Fig. 9, the colour scale is proportional to the density of points. The solid line indicates a linear fit of slope 0.63 . The small empty symbols are the clouds identified in Centaurus A northern filament by Salomé et al. (2017). We observe a clear observational bias: we only detect the clumps with the largest velocity dispersion.

the single dish beam. In order to study the line ratio, we integrated interferometric data within $12^{\prime \prime}$ diameter circles at these three positions. We obtained the spectra shown in Fig. 12. We used the conversion factor $5 \mathrm{Jy} \mathrm{K}^{-1}$ for the $\mathrm{CO}(2-1)$ single dish data. The results of this analysis are presented in Table 5 .

In Table 6, we provide the dust characteristics of the positions, where $\mathrm{CO}(1-0)$ and $\mathrm{CO}(2-1)$ are detected, derived from the maps of Viaene et al. (2014), Draine et al. (2014), and Ford et al. (2013). There is a strong interstellar radiation field $U \sim 20-30$ due to the bulge stellar population, while the cold dust temperature is around $30 \mathrm{~K}$.

Following Eqs. (10) and (11), we estimate a mean luminous mass along the line of sight and within the $12 \operatorname{arcsec}=40 \mathrm{pc}$ beams for these three offsets of $8700 M_{\odot}$. The fraction of gas with respect to the stellar mass along these lines of sight is very small, i.e. $4.0 \times 10^{-4}$, while the dust-to-gas ratio is about 0.01 . This is a sign of high SFR recycling and high metallicity. The Andromeda circum-nuclear region lies well below the dust scaling relation presented by Galliano et al. (2018), as this is observed for early-type galaxies (ETGs).

\subsubsection{Evidence of local non-thermal equilibrium conditions}

The $\mathrm{R}_{21}=\mathrm{CO}(2-1)$-to- $\mathrm{CO}(1-0)$ line ratio (in temperature) was computed for each three detection and is provided in the last column of Table 5. This ratio lies in the range $0.3-0.5$, well below 1 . Hence, we expect this gas to be subthermally excited. This is also a strong evidence that this gas is optically thick. Furthermore, this quite low ratio supports the fact that we have not lost significant flux in the interferometric measurements in these regions detected both in $\mathrm{CO}(1-0)$ and $\mathrm{CO}(2-1)$. We expect that the detected gas is not extended but rather clumpy. We should note that the FWHM velocity width of these lines are in overall good agreement for $\mathrm{CO}(1-0)$ et $\mathrm{CO}(2-1)$, but for MC13-36. In addition, this clump has a velocity mismatch in Li et al. (2019), so it should be considered with caution.

In parallel, there are clouds detected only in $\mathrm{CO}(2-1)$, which fall below our $\mathrm{CO}(1-0)$ sensitivity. There are seven such clouds, six of which have a weak $\mathrm{S} / \mathrm{N}$ of $3 \sigma$ to $4 \sigma$. There is one cloud 

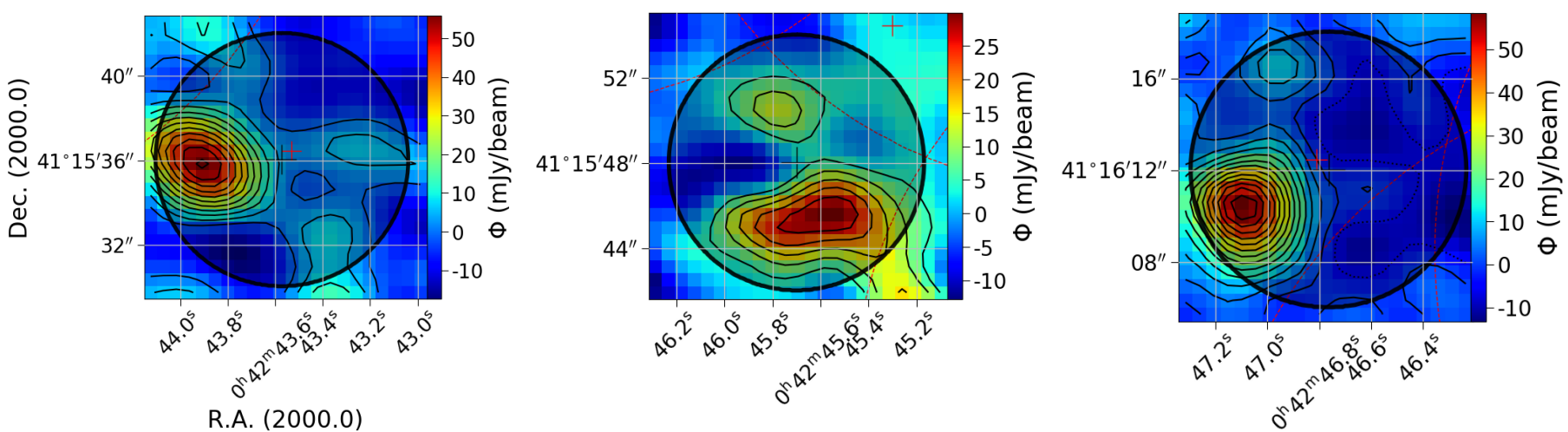

Fig. 11. PdB channels maps associated with the positions listed in Table 5. The darkened region represents beam of the single dish observation from Melchior \& Combes (2013). We notice a position offset and that the whole clump is not always contained in the single dish observations, which explains the missing flux. Velocities obtained with HERA IRAM $30 \mathrm{~m}$ observations correspond to the velocities measured with PdB IRAM interferometric data.

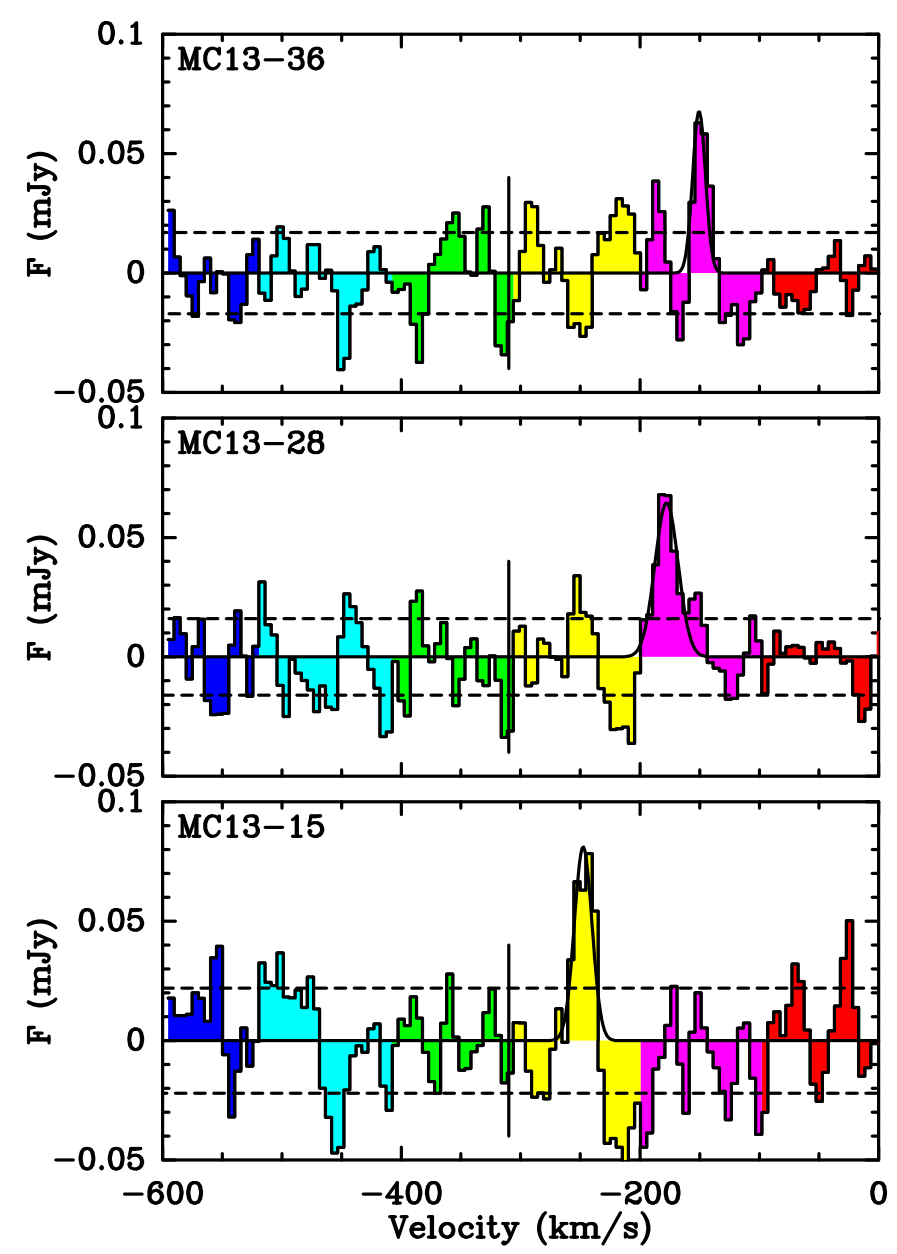

Fig. 12. $\mathrm{CO}(1-0)$ spectra corresponding to the $\mathrm{CO}(2-1)$ detections from Melchior \& Combes (2013, hereafter MC13). The PdB IRAM interferometric data is reprojected on the grid of the data cube obtained with HERA on IRAM $30 \mathrm{~m}$ telescope as described in MC13. We show the spectra in the 12 arcsec beams shown in the Fig. 11. A vertical line indicates the systemic velocity. In each panel, we show the best Gaussian fit used to integrate the CO line, whose results are listed in Table 5.

detected at $6 \sigma$ in $\mathrm{CO}(2-1)$ that is not seen in $\mathrm{CO}(1-0)$ at the offset $\left(-9.0^{\prime \prime},-8.3^{\prime \prime}\right)$. Li et al. (2019) have detected $\mathrm{CO}(3-2)$ molecular gas at this position and estimate a line ratio $R_{32}=0.33 \pm 0.11$. Given the $\mathrm{CO}(1-0) 3 \sigma$ upper limit $\left(S_{\mathrm{CO}}^{1-0}<1.14 \mathrm{Jy} \mathrm{km} \mathrm{s}^{-1}\right)$, we estimate a temperature ratio $R_{2-1 / 1-0} \geq 0.83(3 \sigma)$, which is not very likely according to our modelling, and the $\mathrm{CO}(1-0)$ molecular gas might have been missed owing to interferometric spatial filtering. Relying on the $\mathrm{CO}(2-1)$ and $\mathrm{CO}(3-2)$ single-dish detections, it is probable that this clump has properties similar to the three other clumps analysed below.

The molecular gas is not in thermal equilibrium with the dust. We considered a kinetic temperature in the range $7-15 \mathrm{~K}$ and assumed an excitation temperature $T_{\mathrm{ex}}=5 \mathrm{~K}$. First, assuming this gas is optically thick, we derive a beam filling factor $\eta_{\mathrm{bf}}=T_{\mathrm{obs}} /\left(T_{\mathrm{ex}}-T_{\mathrm{bg}}\right) \times \Delta V / \delta v$, where $T_{\mathrm{obs}}$ is the peak main beam temperature, $T_{\mathrm{bg}}$ is the cosmic microwave background, $\Delta V$ is the FWHM width of the line, and $\delta V$ the velocity resolution. We consider $\mathrm{CO}(2-1)$ measurements as they are not affected by beam filtering. We thus estimate a mean $\eta_{\mathrm{bf}}^{5 K}$ value of $5.2 \%$. This would correspond to clumps with an effective size $d=\sqrt{\eta_{\text {bf }}} D_{\text {Beam }} \simeq 10 \mathrm{pc}$. This value is compatible with the measurements performed in Table 4 and the maps shown in Fig. 11. Second, we derive the mean column density of molecular hydrogen for the three positions studied in this section and found $\left\langle N_{\mathrm{H}}\right\rangle=3 \times 10^{20} \mathrm{~cm}^{-2}$ within the range found for the whole sample. We assumed a standard $\mathrm{CO}$ abundance $N_{\mathrm{CO}}=10^{-4} \times N_{\mathrm{H}}$. We consider as input for the RADEX simulations (van der Tak et al. 2007) a $\mathrm{CO}$ column density of $N_{\mathrm{CO}}^{\prime} / \Delta V=N_{\mathrm{CO}} / \eta_{\mathrm{bf}}^{5 \mathrm{~K}} / \Delta V=$ $1.9 \times 10^{16} \mathrm{~cm}^{-2} \mathrm{~km}^{-1} \mathrm{~s}^{-1}$ considering a mean $\Delta V=30 \mathrm{~km} \mathrm{~s}^{-1}$.

In Fig. 13 we show the line ratios as functions of the hydrogen density. We run RADEX simulations (van der Tak et al. 2007) for three different kinetic temperatures. The densities corresponding to the measured line ratios are weak and imply low excitation temperatures in the range $4-9 \mathrm{~K}$. We find densities that are in the range $60-650 \mathrm{~cm}^{-3}$ (resp. $250-1600 \mathrm{~cm}^{-3}$ ) for $T_{\mathrm{K}}=15 \mathrm{~K}$ (resp. $7.5 \mathrm{~K}$ ). We can further check the consistency of these density estimates. Considering one single effective clump with a mean mass of $8700 M_{\odot}$ and a molecular hydrogen density in the range $60-650 \mathrm{~cm}^{-3}$, we expect a typical size in the range $8-16$ pc. Alternatively, if we consider five clumps in the beam, the clumps have a typical size of $5-10 \mathrm{pc}$.

In Fig. 14, we check that the ratio $R_{21}$ points towards lowdensity values lower than critical for $T_{\mathrm{K}}=15 \mathrm{~K}$. We then derive the $R_{31}$ ratio relying on the $\mathrm{CO}(3-2)$ measurements of $\mathrm{Li}$ et al. (2019). Given the uncertainties, the $R_{31}$ ratios are compatible within $2 \sigma$.

In summary, we detect gas in non-local thermal equilibrium that has a low excitation temperature $\left(T_{\mathrm{ex}} \sim 5-9 \mathrm{~K}\right)$. The kinetic temperature around $15 \mathrm{~K}$ (and possibly smaller) is significantly 
Table 4. Characteristics of the 12 selected molecular clumps.

\begin{tabular}{cccccccc}
\hline \hline ID & $\begin{array}{c}\text { Offsets } \\
(\operatorname{arcsec})\end{array}$ & $\begin{array}{c}R_{\mathrm{eq}} \\
\mathrm{pc}\end{array}$ & $\begin{array}{c}L_{\mathrm{CO}}^{\prime} \\
\mathrm{K} \mathrm{km} \mathrm{s}^{-1} \mathrm{pc}^{2}\end{array}$ & $\begin{array}{c}M_{\mathrm{H}_{2}} \\
10^{3} M_{\odot}\end{array}$ & $\begin{array}{c}\Sigma_{\mathrm{H}_{2}} \\
M_{\odot} \mathrm{pc}^{-2}\end{array}$ & $\alpha_{\mathrm{vir}}$ & $\begin{array}{c}N_{\mathrm{H}_{2}} \\
10^{20} \mathrm{~cm}^{-2}\end{array}$ \\
\hline 1 & $-5.2,-32.5$ & $11.0 \pm 0.7$ & $3321 \pm 138$ & $14.5 \pm 0.6$ & $36.4 \pm 6.3$ & $68 \pm 11$ & $20.2 \pm 3.5$ \\
2 & $30.8,2.3$ & $10.3 \pm 0.9$ & $2202 \pm 137$ & $9.6 \pm 0.6$ & $27.8 \pm 6.5$ & $44 \pm 10$ & $15.4 \pm 3.6$ \\
3 & $34.4,-3.8$ & $15.8 \pm 0.7$ & $2119 \pm 77$ & $9.2 \pm 0.3$ & $11.3 \pm 1.4$ & $121 \pm 15$ & $6.2 \pm 0.8$ \\
4 & $28.9,10.8$ & $12.4 \pm 0.6$ & $1644 \pm 84$ & $7.2 \pm 0.4$ & $14.3 \pm 2.1$ & $71 \pm 13$ & $7.9 \pm 1.2$ \\
5 & $13.1,-22.7$ & $12.4 \pm 0.5$ & $1709 \pm 70$ & $7.5 \pm 0.3$ & $14.9 \pm 1.9$ & $87 \pm 12$ & $8.3 \pm 1.1$ \\
6 & $0.9,-45.9$ & $9.2 \pm 0.7$ & $2940 \pm 107$ & $12.8 \pm 0.5$ & $46.6 \pm 8.4$ & $35 \pm 6$ & $25.9 \pm 4.7$ \\
7 & $19.8,5.9$ & $11.1 \pm 0.5$ & $1087 \pm 49$ & $4.7 \pm 0.2$ & $11.8 \pm 1.7$ & $311 \pm 45$ & $6.6 \pm 0.9$ \\
8 & $35.7,0.4$ & $13.1 \pm 0.8$ & $1112 \pm 65$ & $4.8 \pm 0.3$ & $8.6 \pm 1.6$ & $236 \pm 47$ & $4.7 \pm 0.9$ \\
9 & $2.1,-9.9$ & $17.5 \pm 0.6$ & $650 \pm 30$ & $2.8 \pm 0.1$ & $2.8 \pm 0.3$ & $142 \pm 23$ & $1.5 \pm 0.2$ \\
10 & $7.6,-24.0$ & $19.8 \pm 1.1$ & $968 \pm 41$ & $4.2 \pm 0.2$ & $3.3 \pm 0.5$ & $209 \pm 30$ & $1.8 \pm 0.3$ \\
11 & $-22.3,-33.7$ & $18.0 \pm 0.7$ & $852 \pm 40$ & $3.7 \pm 0.2$ & $3.5 \pm 0.4$ & $66 \pm 12$ & $1.9 \pm 0.2$ \\
12 & $1.5,1.7$ & $17.0 \pm 0.9$ & $579 \pm 37$ & $2.5 \pm 0.2$ & $2.7 \pm 0.4$ & $294 \pm 56$ & $1.5 \pm 0.2$ \\
\hline
\end{tabular}

Notes. The descriptive properties are shown in Table 3. The offsets are calculated according to the optical centre of M31 at J2000 coordinates $00^{\mathrm{h}} 42^{\mathrm{m}} 44.37^{\mathrm{s}}$ and $+41^{\circ} 16^{\prime} 8.34^{\prime \prime}$. The calculation of $R_{\mathrm{eq}}$ is explained in Sect. 2.3. The CO luminosity $L_{\mathrm{CO}}^{\prime}$, luminous mass $M_{\mathrm{H}_{2}}$, surface density $\Sigma_{\mathrm{H}_{2}}$, virial parameter $\alpha_{\mathrm{vir}}$, and $\mathrm{H}_{2}$ column density $N_{\mathrm{H}_{2}}$ calculations are detailed in Sect. 3 .

Table 5. Characteristics of the $\mathrm{CO}(2-1)$ and $\mathrm{CO}(1-0)$ lines integrated in a $12^{\prime \prime}$ FWHM beam at 3 positions.

\begin{tabular}{|c|c|c|c|c|c|c|c|c|c|c|c|c|}
\hline \multirow{3}{*}{$\begin{array}{l}\text { MC13 : Offsets } \\
\text { ID : arcsec }\end{array}$} & \multirow{2}{*}{\multicolumn{2}{|c|}{$\begin{array}{c}S_{\mathrm{CO}} \Delta v \\
\left(\mathrm{Jy} \mathrm{km} \mathrm{s}^{-1}\right) \\
\end{array}$}} & \multirow{2}{*}{\multicolumn{2}{|c|}{$\begin{array}{c}V_{0} \\
\left(\mathrm{~km} \mathrm{~s}^{-1}\right)\end{array}$}} & \multirow{2}{*}{\multicolumn{2}{|c|}{$\begin{array}{c}\Delta V \\
\left(\mathrm{~km} \mathrm{~s}^{-1}\right)\end{array}$}} & \multirow{2}{*}{\multicolumn{2}{|c|}{$\begin{array}{l}S_{\text {peak }} \\
(\mathrm{mJy})\end{array}$}} & \multirow{2}{*}{\multicolumn{2}{|c|}{$\begin{array}{c}\mathrm{rms} \\
(\mathrm{mJy})\end{array}$}} & \multicolumn{2}{|c|}{$(2-1) /(1-0)$} \\
\hline & & & & & & & & & & & $\overline{\text { Flux }}$ & Temp. \\
\hline & $(2-1)$ & $(1-0)$ & $(2-1)$ & $(1-0)$ & $(2-1)$ & $(1-0)$ & $(2-1)$ & $(1-0)$ & $(2-1)$ & $(1-0)$ & Iatro & \\
\hline$-9.0,-$ & $0+0$ & $1.5 \pm 0.3$ & $-248 \pm 2$ & -248.0 & $24 \pm 4$ & $17 \pm 3$ & 115 & 81 & 16 & 7 & 1.41 & $0.35 \pm 0.08$ \\
\hline $28:+15.0,-$ & $8 \pm 0.4$ & $1.6 \pm$ & $0 \pm 1$ & & & 23 & 1 & 6 & 1 & & 2.03 & $0.51 \pm 0.15$ \\
\hline $36:+27.0,+3.7$ & $4.7 \pm 0.8$ & $0.91 \pm 0.2$ & $53 \pm 5$ & -150.0 & $53 \pm 11$ & $13 \pm 3$ & 84 & 68.0 & 20.5 & 17 & 1.23 & $0.31 \pm 0.09$ \\
\hline
\end{tabular}

Notes. The MC13 corresponds to the identification proposed in Melchior \& Combes (2013); the offsets of each position are computed with respect to the optical centre. As the parameters, namely integrated flux $F_{\mathrm{CO}}=S_{\mathrm{CO}} \Delta v$, the central velocity $V_{0}$, linewidth $\Delta V$, peak value $S_{\text {peak }}$, rms level of noise computed on the baseline, and line ratios computed in Jy and in K, are derived from a Gaussian fit in the GILDAS environment.

Table 6. Characteristics derived by Viaene et al. (2014) and Draine et al. (2014) from infrared data at the position of the CO(2-1) and CO(1-0) detections in $36^{\prime \prime} \times 36^{\prime \prime}$ and $18^{\prime \prime} \times 18^{\prime \prime}$ pixels.

\begin{tabular}{|c|c|c|c|c|c|c|c|c|c|c|c|c|c|c|}
\hline \multirow[t]{2}{*}{$\begin{array}{c}\mathrm{MC} 13 \\
\text { ID }\end{array}$} & \multirow[t]{2}{*}{$\begin{array}{l}\eta_{\mathrm{bf}}^{5 \mathrm{~K}} \\
10^{-2}\end{array}$} & \multirow[t]{2}{*}{$\begin{array}{l}\eta_{\mathrm{bf}}^{30 \mathrm{~K}} \\
10^{-3}\end{array}$} & $\begin{array}{c}T_{\mathrm{C}} \\
\mathrm{K} \\
\end{array}$ & $\begin{array}{c}T_{\mathrm{W}} \\
\mathrm{K}\end{array}$ & $\begin{array}{c}M_{\text {dust }} \\
M_{\odot}\end{array}$ & $\begin{array}{c}M_{\text {star }} \\
10^{7} M_{\odot}\end{array}$ & $\tau_{V}^{\text {dust }}$ & $\tau_{V}^{\text {Diff }}$ & $\begin{array}{c}\text { SFR } \\
M_{\odot} \mathrm{Myr}^{-1} \\
\end{array}$ & $U_{\text {mean }}$ & $U_{\min }$ & $\begin{array}{c}T_{\text {mean }} \\
\mathrm{K}\end{array}$ & $\begin{array}{c}T_{\min } \\
\mathrm{K}\end{array}$ & \multirow{2}{*}{$\begin{array}{c}\mathrm{SFR} \\
M_{\odot} \mathrm{Myr}^{-1} \\
(\mathrm{~F} 13)\end{array}$} \\
\hline & & & \multicolumn{7}{|c|}{ (Viaene et al. 2014) } & \multicolumn{4}{|c|}{ (Draine et al. 2014) } & \\
\hline 15 & 4.8 & 4.0 & & & 33 & 1.8 & $\Omega_{1}$ & 0 & & & 15.0 & & & 8 \\
\hline 28 & 3.1 & 2.7 & 29.8 & 63.7 & 33 & 1.8 & 0.152 & 0.022 & 0.22 & 32.3 & 22.0 & 32.1 & 30.13 & 77 \\
\hline 36 & 7.8 & 6.6 & 29.8 & 63.7 & 38 & 2.0 & 0.162 & 0.027 & 3.25 & 27.1 & 25. & 31.2 & 30.7 & 133 \\
\hline
\end{tabular}

Notes. As described in Sect. 4.1 and in Melchior \& Combes (2016), $\eta_{\text {bf }}$ provides the beam filling factor computed for a brightness temperature of $5 \mathrm{~K}$ relying on the RADEX results presented in Sect. 4.1 and $30 \mathrm{~K}$ assuming it is thermalised with the dust. The values $T_{\mathrm{C}}$ and $T_{\mathrm{W}}$ correspond to the cold and warm dust components; $M_{\text {dust }}$ and $M_{\text {star }}$ are the dust and stellar masses scaled to the prorata of the $12^{\prime \prime}$ CO FWHM beam; and $\tau_{V}^{\text {dust }}$ and $\tau_{V}^{\text {Diff }}$ are the dust and diffuse dust optical depths, given for the order of magnitude as they are the poorest constrained parameters according to Viaene et al. (2014). The SFR is scaled to the pro rata of the $12^{\prime \prime}$ CO FWHM beam. The last column provides the SFR estimate of Ford et al. (2013) integrated on the $12^{\prime \prime}$ beam.

weaker that the dust temperature estimated in the infrared around $30 \mathrm{~K}$. The volume density of molecular gas is well below the critical density.

\subsection{Tracing star formation}

While the central region of Andromeda does not reveal any obvious star forming region, global star formation estimates usually display a non-zero SFR in this region known to host ionised gas and dust. Depending on the star formation tracers, quite differ- ent values have been estimated. These different values however do not affect the global (all galaxy) SFR estimates (Viaene et al. 2014; Ford et al. 2013), since the SFR is negligible in this central region. We note that the bulge is also often avoided (e.g. Lewis et al. 2015, 2017).

In distant galaxies, it is customary to estimate the amount of obscured star formation observed in FUV by correcting the observed emission from young stars with the observed $24 \mu \mathrm{m}$ Spitzer flux. This was studied statistically by Leroy et al. (2008) and on sub-kiloparsec scale by Bigiel et al. (2008), 

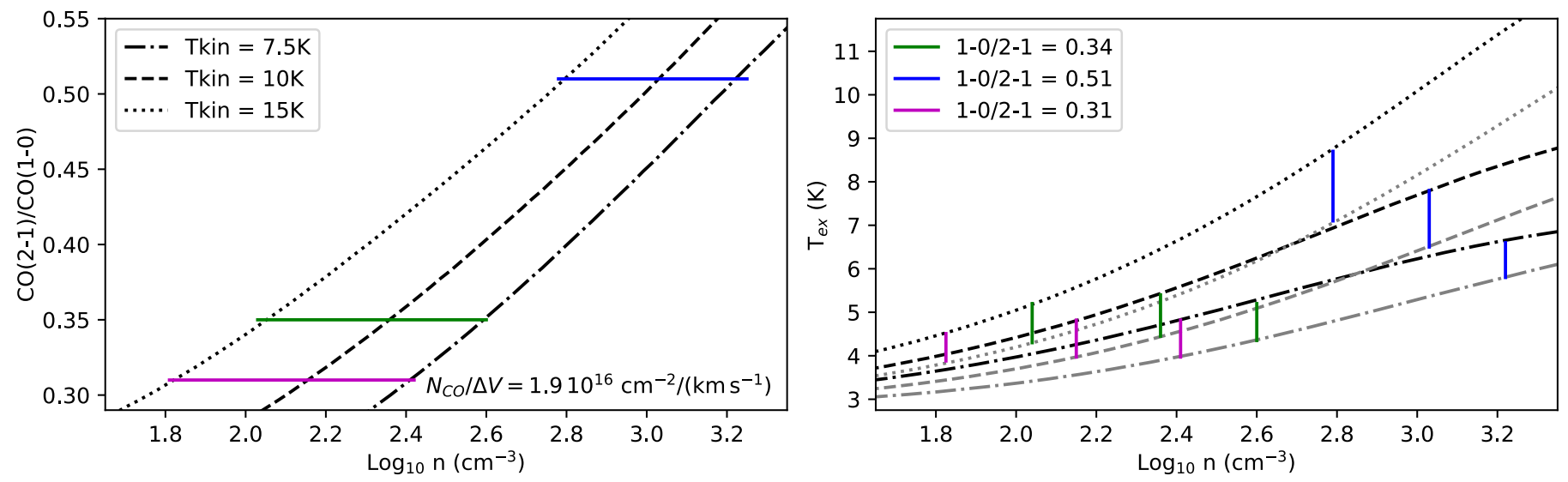

Fig. 13. RADEX simulations. We consider a standard CO abundance $N_{\mathrm{CO}}=10^{-4}\left\langle N_{\mathrm{H}_{2}}\right\rangle$. We measure a mean molecular hydrogen column density of $3 \times 10^{20} \mathrm{~cm}^{-2}$ that we correct for the surface beam filling factor $\left(\eta_{\mathrm{bf}}=0.052\right)$ assuming the $\mathrm{CO}(2-1)$ is optically thick and computed for $T_{\mathrm{ex}}=5 \mathrm{~K}$. Last, we estimate a mean $\Delta V$ of $30 \mathrm{~km} \mathrm{~s}^{-1}$. Left panel: the different line types represent the simulations for 3 different kinetic temperature $T_{\text {kin }}$. Left and right panels: expected $\mathrm{CO}(2-1)$ to $\mathrm{CO}(1-0)$ line ratio (in temperature) and excitation temperature of the 2 lines, respectively, as a function of the hydrogen density. Right panel: the black (resp. grey) lines correspond to the $\mathrm{CO}(1-0)$ (resp. $\mathrm{CO}(2-1)$ ) line. The green, blue, and magenta ticks correspond to the line ratios measured in the 3 positions. This shows that this gas is subthermally excited and well below the expected critical density for a kinetic temperature of $10 \mathrm{~K}, n_{\text {crit }}^{1-0}=2.2 \times 10^{3} \mathrm{~cm}^{-3}$ and $n_{\text {crit }}^{2-1}=9.7 \times 10^{3} \mathrm{~cm}^{-3}$.

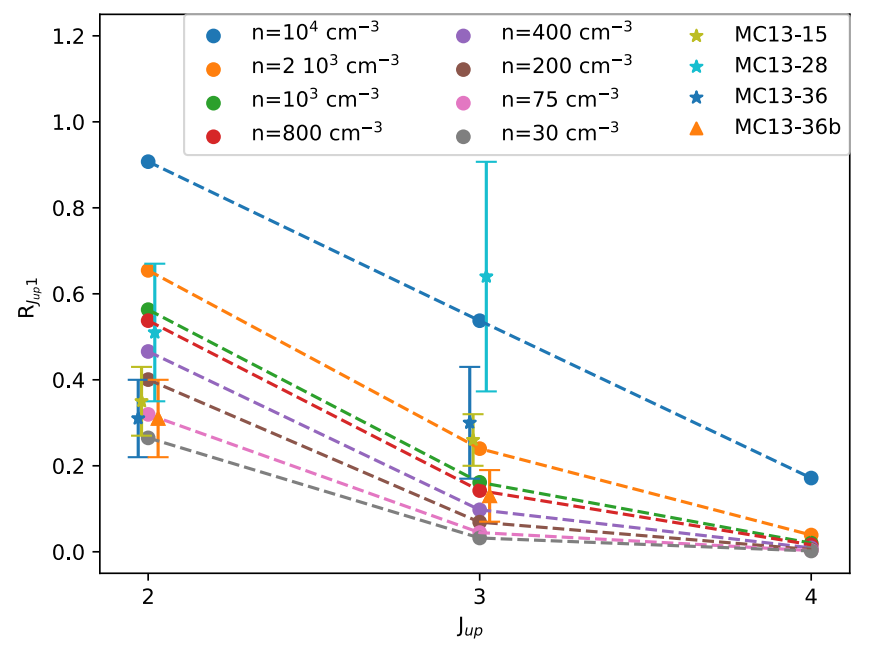

Fig. 14. Comparison of the $\mathrm{CO}$ line ratios observed for 3 positions of the circum-nuclear disc with RADEX simulations. As discussed in the text, we ran RADEX simulations assuming a large velocity gradient with a kinetic temperature of $15 \mathrm{~K}$ and $N_{\mathrm{CO}} / \Delta V=1.9 \times 10^{16} \mathrm{~cm}^{-2} \mathrm{~km}^{-1} \mathrm{~s}$. The bullets indicate the results of these simulations for different volume density (n). We superimposed the $R_{21}$ line ratios computed in Table 5, and $R_{31}$ ratio derived from the $\mathrm{CO}(3-2)$ James Clerk Maxwell Telescope observations of Li et al. (2019). For the positions 15 and 28, there is a good velocity agreement, while Li et al. (2019) have found a different $\mathrm{CO}(3-2)$ velocity for the position 36 , when compared to our measurements. This is why there are 2 points corresponding to this position. The measurement uncertainties are shown for each point. Interestingly, most $R_{21}$ points are incompatible with a critical density and the $R_{32}$ points at $2 \sigma$.

and applied to Andromeda by Ford et al. (2013). The FUV maps are expected to trace stars up to 75-100 Myr old. It can be severely obscured by dust, while the UV emission from young stars is expected to heat and re-radiate in the mid-infrared. The idea is hence to remove the contribution from the stellar emission and to perform a linear combination of the FUV and $24 \mu \mathrm{m}$ fluxes calibrated as proposed originally by Salim et al. (2007) and further tested by Leroy et al. (2008).
The region studied in this work is about one-tenth of the region studied as the bulge in Ford et al. (2013). It is dominated by the stellar light with a very strong gradient. In this section, we reinvestigate this estimation of the SFR in the region where we observed molecular gas. In Sect. 4.2.1, we gather and reproject a series of publicly available FUV-to- $24 \mu \mathrm{m}$ images to study the spectral energy distribution of this region. In Sect. 4.2.2, we compare the spectral energy distribution with various templates. On this basis, we show that an estimate of the SFR based on FUV and $24 \mu \mathrm{m}$ emissions corrected from stellar emission detect features at this noise level.

\subsubsection{Spectral energy distribution}

We gathered photometric maps obtained in the past ten years on the Andromeda galaxy. While we are interested in the central kiloparsec field, it becomes important to get a full mosaic of the galaxy as the background determination is challenging and residuals prevent any photometric use of the maps. In the UV, we used the FUV and NUV GALEX Atlas of Nearby Galaxies (Gil de Paz et al. 2007). We retrieve 1 square degree ugriz SDSS mosaic from Image Mosaic Service (Montage) at IPAC. We get the JHK 2MASS Large Galaxy Atlas (Jarrett et al. 2003). We retrieve IRAC $3.6 \mu \mathrm{m}$ and $4.5 \mu \mathrm{m}$ and MIPS $24 \mu \mathrm{m}$ maps from Spitzer Heritage Archives. The IRAC $5.8 \mu \mathrm{m}$ and $7.9 \mu \mathrm{m}$ maps suffer from bad background estimates in the central kiloparsec and we were not able to use them ${ }^{1}$. Last, we used the WISE image services to get the $3.4 \mu \mathrm{m}$ and $4.6 \mu \mathrm{m}$ maps found in excellent agreement with the IRAC Spitzer maps obtained at similar wavelengths.

In order to study the spectral energy distribution on a pixel basis, we first convolved each map to a 7 arcsec FWHM Gaussian relying on the convolution technique based on kernels proposed by Aniano et al. (2011). Second, we reprojected all the maps on a 2.4 arcsec grid corresponding to $24 \mu \mathrm{m}$ Spitzer maps with Kapteyn Package. We then plotted the spectral energy distribution for each pixel and/or in an integrated area. To compute the spectral energy distribution shown in Figs. 15

1 We also have to remove a constant background of $2.5 \mathrm{MJy} \mathrm{sr}^{-1}$ to get results that are consistent with Ford et al. (2013). 


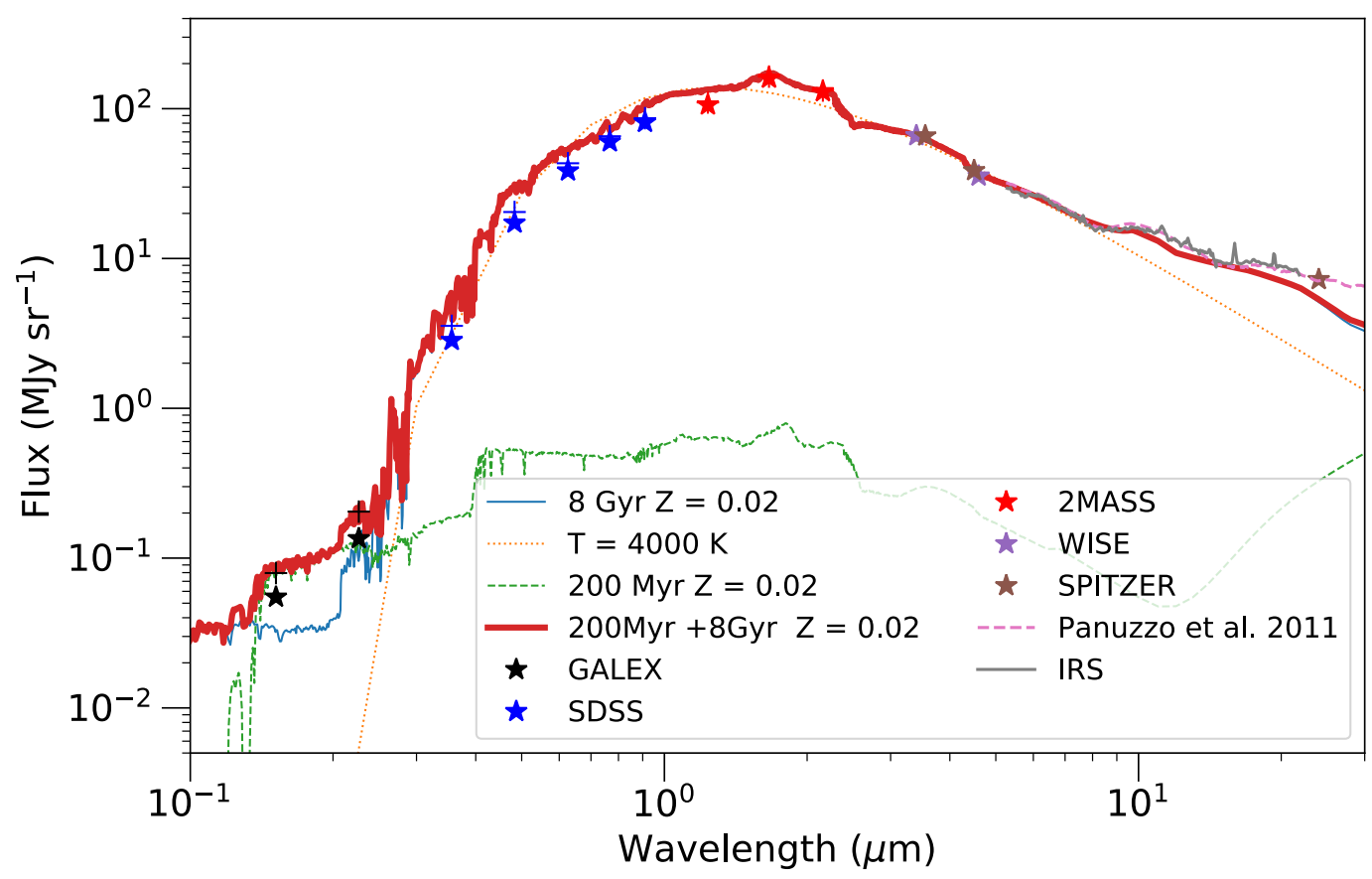

Fig. 15. Spectral energy distribution integrated over the region observed with IRAM-PdB, excluding the region with the UV bright nuclear disc (Lauer et al. 2012). As described in Sect. 4.2.1, we integrate over this region each photometric map available in this region after being reprojected on the $24 \mu \mathrm{m}$ Spitzer map and convolved to a 7 arcsec FWHM Gaussian (Aniano et al. 2011). The star symbols correspond to the values thus achieved for each survey, while the crosses represent the same measurements after a Galactic extinction correction $(E(B-V)=0.05)$. The integrated IRS spectra from Hemachandra et al. (2015) and the passive template from Panuzzo et al. (2011) are shown in grey. Last, we superimpose different stellar templates from Bressan et al. (1998) and Fioc \& Rocca-Volmerange (1999), as well as a $4000 \mathrm{~K}$ black body. We show that GALEX points support solar metallicity templates, with a small contribution of a young stellar population (200 Myr).

and 16 , we reproject the maps on the nuclear region observed by Hemachandra et al. (2015) with Spitzer/Infrared Spectrograph (IRS). The nucleus was observed with the IRS short-low (SL1, SL2 and SL3) and long-low (LL2) modules, which allows observations over the 5.2-20.75 $\mu \mathrm{m}$ band. The maps sizes were obtained by 18 overlapping observations for SL $\left(32^{\prime \prime} \times 57^{\prime \prime}\right)$ and 11 overlapping observations for $\operatorname{LL}\left(58^{\prime \prime} \times 168^{\prime \prime}\right)$, both regions overlapping in the central area (see Fig. 17). The spectrum was integrated over the central region from which we cropped the sides since they contained clear border effects, including strong negative signals. The SL2 $(5.2-7.6 \mu \mathrm{m})$ and SL1 (7.5$14.5 \mu \mathrm{m})$ maps were connected using the overlapping SL3 (7.33$8.66 \mu \mathrm{m})$ mean flux. The SL3 map showed no offset with SL2, so the offset was added to SL1. The SL1 map was connected to LL2 $(14.5-20.75 \mu \mathrm{m})$ with an offset to LL2 to obtain a coherent continuity with Spitzer IRAC $(4.5 \mu \mathrm{m})$ and MIPS $(24 \mu \mathrm{m})$ measurements.

Figure 15 corresponds to the field of view observed with IRAM-PdB, but excluding the FUV bright region (with a FUV flux non-corrected for Galactic extinction smaller than $0.15 \mathrm{MJy} \mathrm{sr}^{-1} \mathrm{kpc}^{-2}$ ). We find a good agreement with an 8 Gyr stellar templates from Bressan et al. (1998) and a small contribution from a $200 \mathrm{Myr}$ stellar population (PEGASE.2; Fioc \& Rocca-Volmerange 1999). The crosses include a correction of foreground extinction (Fitzpatrick 1999) assuming $E(B-V)=0.05$ following (e.g. Dalcanton et al. 2012) and $R_{V}=3.1$. This is in general agreement with the current belief that this region is dominated by an old stellar population (e.g. Rosenfield et al. 2012). In the near-infrared, we observe an excellent agreement with all stellar templates below $5 \mu \mathrm{m}$. We find an excellent agreement between the near-infrared measurements and the Hemachandra et al. (2015) IRS Spitzer spectra; there is a significant silicate "bump" around $10 \mu \mathrm{m}$. This spectral energy distribution is also very close to the passive galaxy template shown in Fig. 2 of Panuzzo et al. (2011). This is typical of ETGs as discussed by Panuzzo et al. (2011) and Rampazzo et al. (2013) and more generally old stellar populations. While Saglia et al. (2010) observed a metallicity gradient with slit spectroscopy but with a large uncertainty, metal-rich $Z=0.05 \leq 10 \mathrm{Gyr}$ templates from Bressan et al. (1998) exhibit an infrared excess that is incompatible with the $24 \mu \mathrm{m}$ Spitzer data. Solar metallicity SSP templates of $8 \mathrm{Gyr}$ and $200 \mathrm{Myr}$ adjust the data correctly. Large metallicity templates improve the match with the data in the optical part but not in the UV nor IR (for an age smaller or equal to $10 \mathrm{Gyr}$ ). One can note the presence of weak fine-structure emission lines discussed in Hemachandra et al. (2015), which confirms the presence some interstellar ionised gas. One can point to relatively large [NeIII] emission line, often correlated to the $24 \mu \mathrm{m}$ dust emission (e.g. Inami et al. 2013).

Figure 16 is restricted to the FUV bright region excluded in Fig. 15. This region is known to host an UV bright $200 \mathrm{Myr}$ stellar cluster as studied by Lauer et al. (2012). It is more difficult to match the data with a single template, suggesting a mixture of stellar population from different origins. The contribution of the $200 \mathrm{Myr}$ is more important, and it is also necessary to add a large metallicity template to reproduce the UV and optical part of the spectral energy distribution. In this case, the stellar background is stronger and the fine-structure emission lines are relatively weaker. Again, the FUV emission can be accounted for by stellar templates. There is no sign of star formation, while some atomic ionised gas and weak dust emission (see below) are tracing the interstellar medium of this region, probably heated by the stellar population (Groves et al. 2012). 


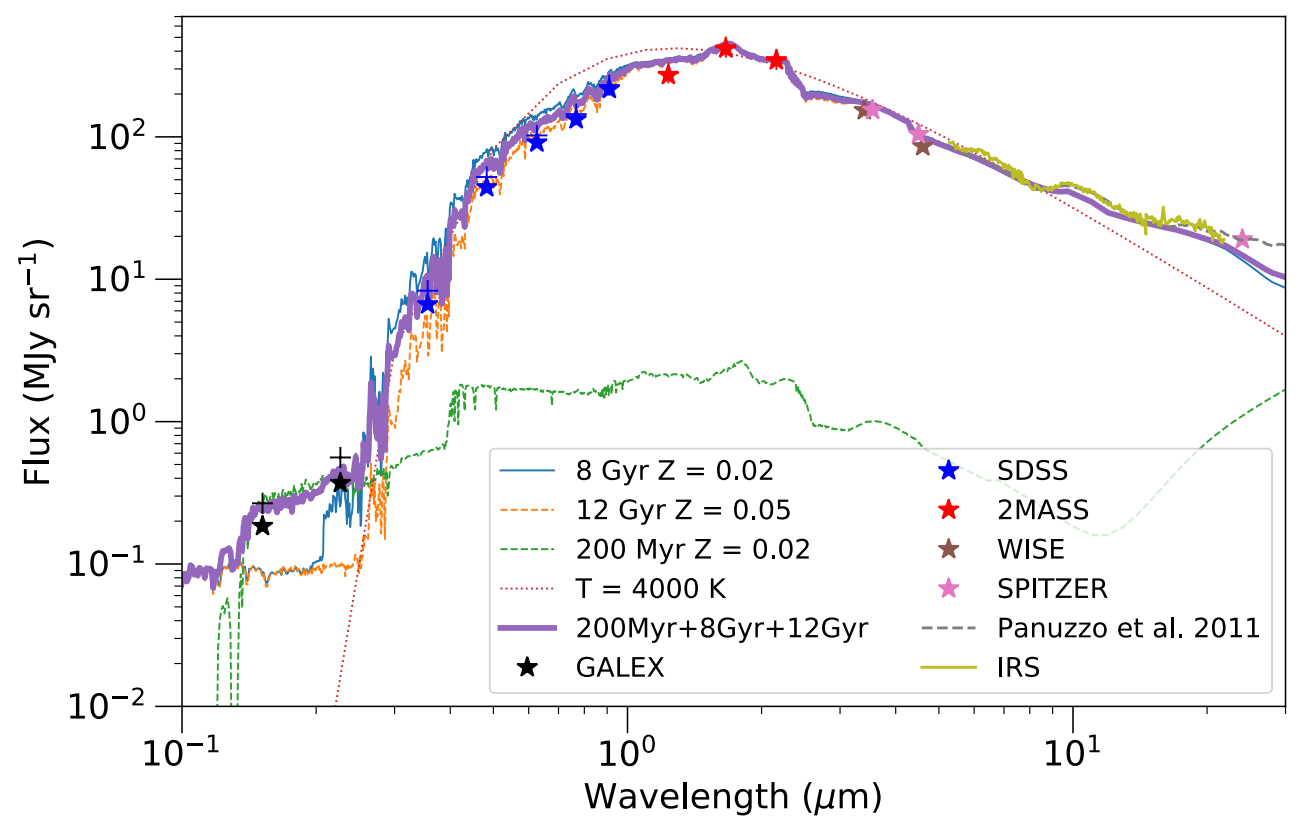

Fig. 16. Spectral energy distribution integrated over the nuclear disc containing an FUV bright excess (with an FUV flux non-corrected for Galactic extinction larger than $0.15 \mathrm{MJy} \mathrm{sr}^{-1} \mathrm{kpc}^{-2}$ ). The same data sets and template library as in Fig. 15 are used. In this case, GALEX points are more difficult to reproduce. Aside from the need for a larger $200 \mathrm{Myr}$ stellar population contribution, a fraction of a 12 Gyr large metallicity template is required in addition to the $8 \mathrm{Gyr}$ solar metallicity template.

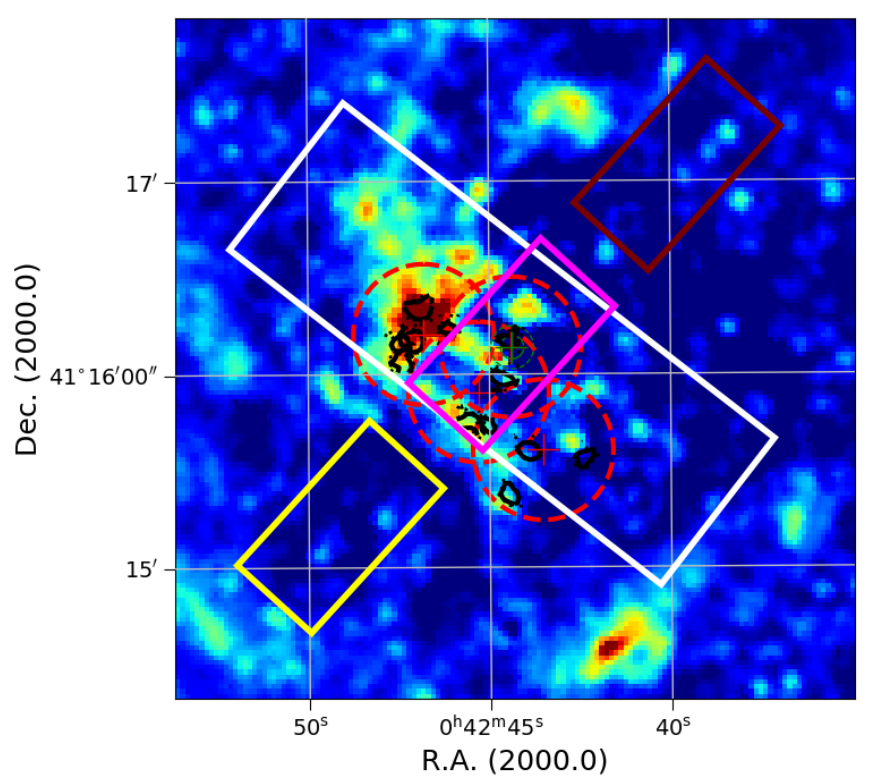

Fig. 17. Map of $\Sigma_{\mathrm{SFR}}$ from Ford et al. (2013) on which we show the regions observed with Spitzer/IRS by Hemachandra et al. (2015) with rectangles. Each rectangle corresponds to a region observed by a different module of Spitzer/IRS. The white rectangle corresponds to the LL2 map, the red indicates SL2, the yellow indicates SL1, and the magenta shows an overlapped region between both SL1 and SL2. The dashed red circles indicate the fields of our data cube and the black contours correspond to the selected clumps.

\subsubsection{Dust emission and star formation estimate}

Leroy et al. (2013) used a SFR estimate based on FUV and $24 \mu \mathrm{m}$. This method was used by Ford et al. (2013) who seem to overestimate the SFR in the circum-nuclear region with respect to (e.g. Viaene et al. 2014). In Fig. 18, we compute the correlation between the FUV and $24 \mu \mathrm{m}$ with $3.6 \mu \mathrm{m}$ as computed by Ford et al. (2013); in this figure, the colour-coding corresponds to the projected distance to the centre. The latter authors used the $3.6 \mu \mathrm{m}$ as an estimate of the stellar contribution to the $24 \mu \mathrm{m}$ and FUV for a $3.6 \mu \mathrm{m}$ flux smaller than
$100 \mathrm{MJy} \mathrm{sr}^{-1}$. We note that there is a non-linear increase for a $3.6 \mu \mathrm{m}$ flux above $100 \mathrm{MJy} \mathrm{sr}^{-1}$ observed both in FUV and at $24 \mu \mathrm{m}$. According to the spectral energy distribution of the region shown in Fig. 16, this can be accounted for by a mixture of stellar populations.

Figure 19 represents the $24 \mu \mathrm{m}$ and FUV emissions after the subtraction of the emission from the stellar population performed with the adjustments shown in Fig. 18. We detect in the $24 \mu \mathrm{m}$ map dust emission in the east side of the IRAM$\mathrm{PdB}$ observations corresponding to three high $S N R_{\text {tot }}(>45)$ CO clumps, as shown in Fig. 7, and corresponding to the MC13 \#28 and \#36 positions, $\mathrm{CO}(2-1)$ was detected. We note that weak $11.2 \mu \mathrm{m}$ is detected in this region observed at the edge of the IRS/Spitzer field (Hemachandra et al. 2015). In addition, a $24 \mu \mathrm{m}$ clump is observed $15^{\prime \prime}$ north of the nucleus, where weak $11.2 \mu \mathrm{m}$ and $17 \mu \mathrm{m}$ PAH features are detected. No molecular gas has been detected there. In these positions, no 6-9 $\mu \mathrm{m}$ PAH features escape detection even though Hemachandra et al. (2015) discuss the importance of template subtractions. However, such 6-9 $\mu \mathrm{m}-11.2 \mu \mathrm{m}$ PAH contrast is often observed in elliptical galaxies (Kaneda et al. 2005; Vega et al. 2010). Aside from a very weak feature next to the nucleus, the other $24 \mu \mathrm{m}$ features are at the level of noise. In FUV, there is a bright spot next to the centre; this spot most probably corresponds to the subtraction of the FUV stellar cluster, while there might be some extinction on the north-west side of the field, corresponding to the inclination of the main disc. These features correspond to about $10 \%$ of the stellar continuum in this region and their amplitude is very sensitive to the linear correction adopted (see Fig. 18). When we combined these two maps following Leroy et al. (2008), we obtain the SFR map on the left-hand side of Fig. 20. This map seems dominated by the dust emission discussed above, which is most probably heated by the interstellar radiation field (Groves et al. 2012). While this map averages to zero with a standard deviation of $0.004 M_{\odot} \mathrm{yr}^{-1}$, the correlation between the FUV and $24 \mu \mathrm{m}$ maps represented in Fig. 19 is shown in the right panel of Fig. 20. Leroy et al. (2008) explained that in case of star formation, a correlation is expected between the $24 \mu \mathrm{m}$ dust emission and the FUV extinction. We note that we observe a correlation in the north-west side, corresponding to the PAH dust 

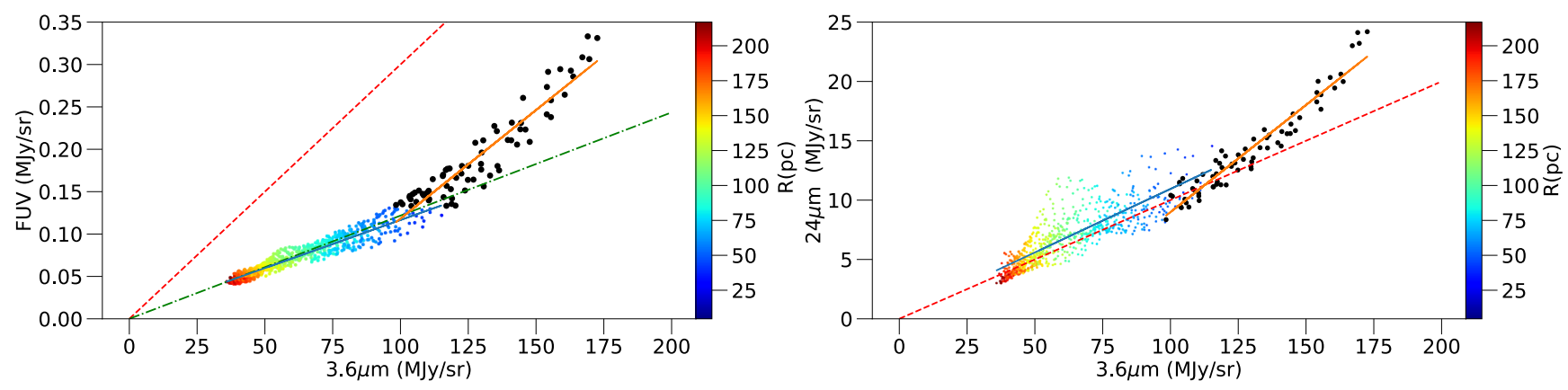

Fig. 18. Correlations of the FUV and $24 \mu \mathrm{m}$ flux with the $3.6 \mu \mathrm{m}$ flux in the field of view observed with IRAM-PdB. The colour coding corresponds to the radial distance to the centre. The black large points correspond to measurements with a FUV flux (non-corrected for Galactic extinction) larger than $0.09 \mathrm{MJyr} \mathrm{sr}^{-1}$. The red dashed lines correspond to the correction used by Leroy et al. (2013); the green dashed line indicates the correction used by Ford et al. (2013). The blue and orange lines correspond to the linear adjustments performed on the two regions and tentatively used to correct the FUV and $24 \mu \mathrm{m}$ fluxes from stellar population.
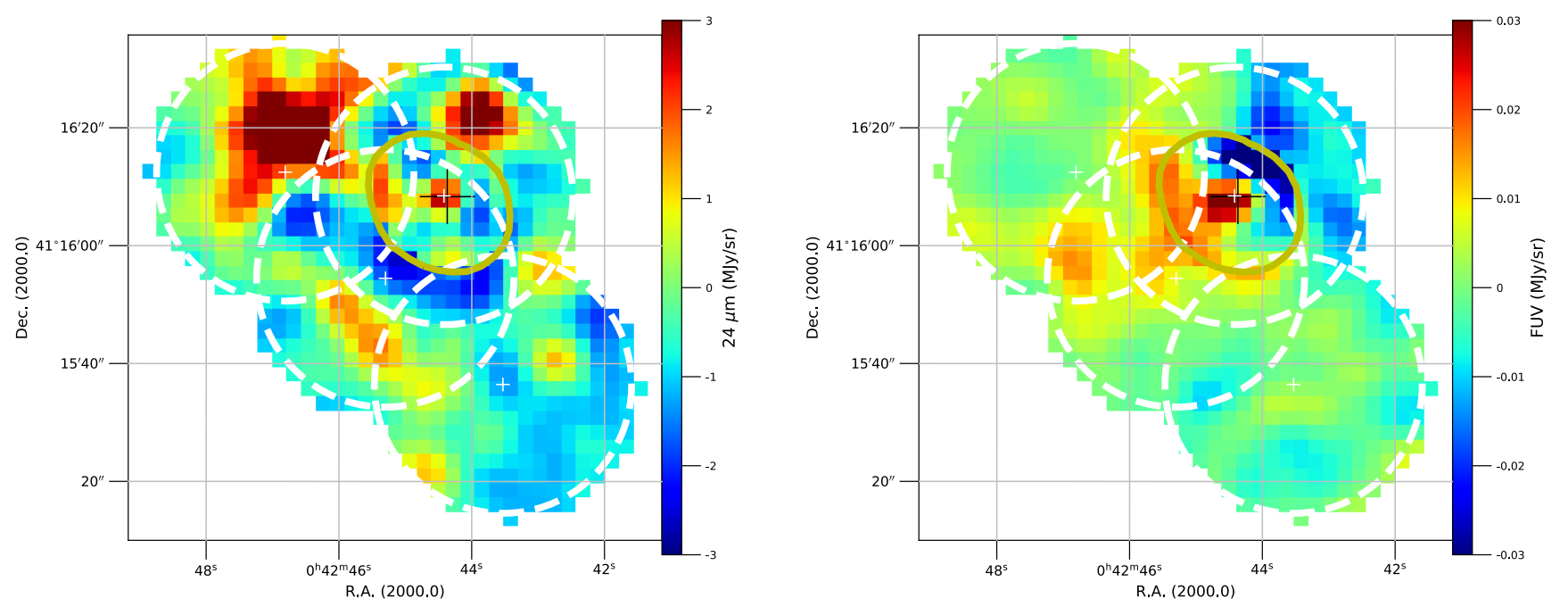

Fig. 19. Different components used in star formation estimates. Following Leroy et al. (2008) and Ford et al. (2013), we subtract the stellar component on the $24 \mu \mathrm{m}$ Spitzer map (left panel) and on FUV GALEX map (right panel). The positions of the IRAM-PdB interferometer CO(1-0) observations are shown with white dashed circles. The yellow contour corresponds to the FUV flux (non-corrected for Galactic extinction) of $0.09 \mathrm{MJyr} \mathrm{sr}^{-1}$.
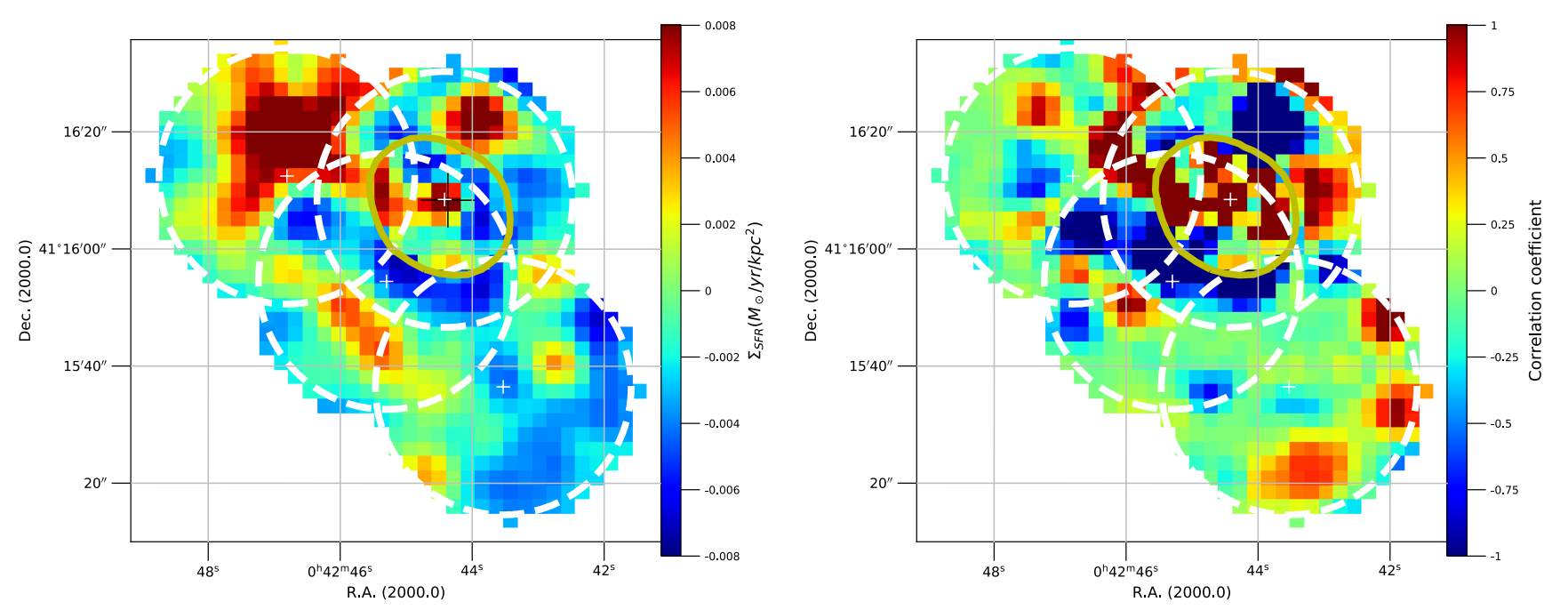

Fig. 20. Star formation estimate derived the FUV and $24 \mu \mathrm{m}$ components indicated in Fig. 19 (left panel) and the correlation factor between these two components (right panel). The positions of the IRAM-PdB interferometer $\mathrm{CO}(1-0)$ observations are shown with white dashed circles. The yellow contour corresponds to a FUV GALEX flux (non-corrected for Galactic extinction) of $0.09 \mathrm{MJy} \mathrm{sr}^{-1}$. 


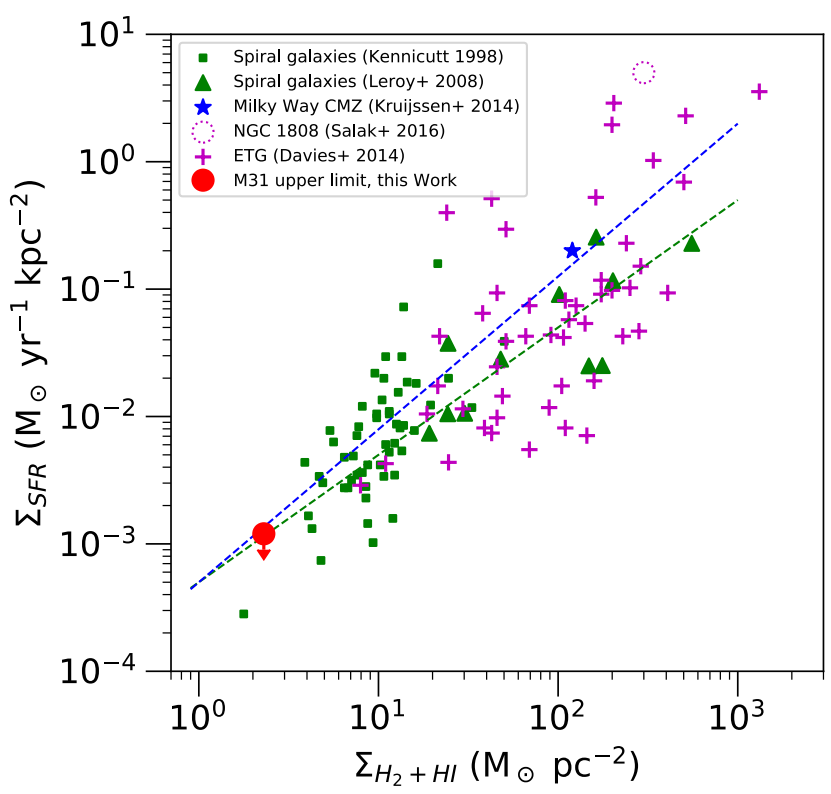

Fig. 21. Surface density of SFR with respect to surface density of $\mathrm{H}_{2}+\mathrm{HI}$ gas for various galaxies. The red filled circle denotes the M 31 circum-nuclear region, where $\Sigma_{\mathrm{H}_{2}+\mathrm{HI}}$ was calculated from our molecular gas measurements, the estimated surface density of $\mathrm{HI}$ in the corresponding region of M31 from Braun et al. (2009) (between 1 and $\left.2 M_{\odot} \mathrm{pc}^{2}\right)$, and $\Sigma_{\mathrm{SFR}}$ from our FUV plus $24 \mu \mathrm{m}$ map shown in the left panel of Fig. 20. The green triangles indicate the central region of spiral galaxies from Leroy et al. (2008). The magenta dotted circle indicates the circum-nuclear region $(r<200 \mathrm{pc})$ of NGC 1808 with $\Sigma_{\mathrm{H}_{2}}$ from Salak et al. (2016) and $\Sigma_{\mathrm{SFR}}$ estimated from Krabbe et al. (1994). The magenta crosses indicate the central parts of ETGs from Davis et al. (2014). The green squares indicate spiral galaxies from Kennicutt (1998). The green square close to M 31 denotes NGC 4698, an early-type spiral with a ring-like disc of star formation, and a decoupled nuclear disc (Corsini et al. 2012). The green dashed line indicates the Kennicutt-Schmidt law with a slope $N=1.0$, and the blue dashed line indicates the linear regression applied on the whole sample.

emission discussed above, which supports the presence of dust with no molecular gas detection. There is also a correlation in the middle of the field, but it corresponds to a FUV excess and negative dust feature. This correlation is possibly an artefact at the limit $\left(0.09 \mathrm{MJy} \mathrm{sr}^{-1}\right.$ in FUV flux) used to correct the stellar contribution and does not correspond to an effect of a star forming region. The regions in which we detect molecular gas do not exhibit a correlation coefficient close to -1 , as can be seen in a comparison of Fig. 7 with the right panel of Fig. 20. This supports the view that there is residual gas in this region that is not forming stars, and some dust is heated by the intermediate and old stellar population.

\subsection{Kennicutt-Schmidt law}

M31 quiescence in its central region is made strikingly clear in our measurements. We estimate in the previous section an upper limit on the surface density SFR $\Sigma_{\mathrm{SFR}}<0.0012$ $(3 \sigma) M_{\odot} \mathrm{yr}^{-1} \mathrm{kpc}^{-2}$. In Table 4 , we estimate a mean molecular gas surface density of the detected clumps $\Sigma_{\mathrm{H}_{2}}=15 M_{\odot} \mathrm{pc}^{-2}$, but when averaged on the region (of equivalent radius of $165 \mathrm{pc}$ ), this density reduces to $\Sigma_{\mathrm{H}_{2}}=0.9 M_{\odot} \mathrm{pc}^{-2}$. This is a lower limit as smaller clumps have escaped detection. Last, we rely on Braun et al. (2009) to estimate a neutral hydrogen surface density of the region of $\Sigma_{\mathrm{HI}}=2 \pm 0.5 M_{\odot} \mathrm{pc}^{-2}$. In Fig. 21, we compare to the Kennicutt-Schmidt law $(N=1.4)$ with data from Williams et al. (2018), Ford et al. (2013), Bigiel et al. (2010), and Leroy et al. (2008). Our upper limit is compatible with surface density SFR derived from Ford et al. (2013) and Viaene et al. (2014).

The circum-nuclear region of Andromeda is typically not forming stars and we reach the limits as discussed by Calzetti et al. (2012). It can be argued that the region considered is small but we nevertheless integrate along the line of sight given the inclination of the disc.

Following Leroy et al. (2008), we define star formation efficiency (SFE) as the SFR surface density per unit $\mathrm{H}_{2}$, $\operatorname{SFE}\left(\mathrm{H}_{2}\right)=\Sigma_{\mathrm{SFR}} / \Sigma_{\mathrm{H}_{2}}$. Hence, we find a SFE of $\mathrm{H}_{2}$ smaller than $1.3 \times 10^{-9} \mathrm{yr}^{-1}(3 \sigma)$, which is within the uncertainties of Leroy et al. (2008) who found $\operatorname{SFE}\left(\mathrm{H}_{2}\right)=5.25 \pm 2.5 \times 10^{-10} \mathrm{yr}^{-1}$ at scales of $800 \mathrm{pc}$. A constant $\operatorname{SFE}\left(\mathrm{H}_{2}\right)$ is expected if the GMC properties are universal and the conditions within the GMC constitute the driving factor (Krumholz \& McKee 2005).

\section{Conclusions}

We have analysed molecular observations made with the IRAM Plateau de Bure interferometer, towards the central $250 \mathrm{pc}$ of M31. The $\mathrm{CO}(1-0)$ mosaic was compared with previous $\mathrm{CO}(2-$ 1) single dish mapping with the IRAM $30 \mathrm{~m}$ HERA instrument. We studied the spectral energy distribution of the region and tracers of star formation. The main results can be summarised as follows:

1. We first identified molecular clumps from high $\mathrm{S} / \mathrm{N}$ peaks in the map and selected genuine clouds using both robust data analysis and statistical methods. Our modified approach to the CPROPS algorithm allowed us to extract a catalogue of $12 \mathrm{CO}(1-0)$ clumps with a total $\mathrm{S} / \mathrm{N}$ larger than 15 in a region of low CO density.

2. We derived the size, velocity dispersion, $\mathrm{H}_{2}$ mass, surface density, and virial parameter for each cloud. The clouds follow the Larson's mass-size scaling relation, but lie above the velocity-size relation. We suggest that they are not virialised, but might be the superimposition of smaller entities.

3. We measured a gas-to-stellar mass ratio of $4 \times 10^{-4}$ in this region.

4. We discussed the $\mathrm{CO}(2-1)$-to- $\mathrm{CO}(1-0)$ line ratio from the single dish observations from Melchior \& Combes (2013) when the $\mathrm{CO}(1-0)$ map is smoothed to the 12 arcsec resolution. We were able to compute the $\mathrm{CO}(2-1)$-to- $\mathrm{CO}(1-0)$ line ratio for three positions. This ratio is below 0.5 , supporting non-LTE conditions. Relying on RADEX simulations, we discuss that this optically thick gas has a low gas density in the range $60-650 \mathrm{~cm}^{-3}$ with subthermal excitation $\left(T_{\mathrm{ex}}=5-9 \mathrm{~K}\right.$. We find a filling factor of order $5 \%$.

5. We studied the spectral energy distribution of the region, and show that it is compatible with a quiescent elliptical galaxy template that has weak mid-infrared, atomic fine-structure emission lines. These lines correlates with the $24 \mu \mathrm{m}$ dust emission detected in the region.

6. We observed correlation between the FUV extinction and $24 \mu \mathrm{m}$ dust emission in a position at 15 arcsec north of the nucleus, where Hemachandra et al. (2015) detected 11.2 and 17. $\mu \mathrm{m}$ PAH. No molecular gas is detected in this position. In the region located east of the nucleus, where several molecular clouds were detected, there is $24 \mu \mathrm{m}$ dust emission and 11.2 $\mu \mathrm{m}$ PAH emission (Hemachandra et al. 2015).

7. We subtracted the stellar contribution in order to use $24 \mu \mathrm{m}$ and FUV as tracers of star formation. This region averages 
to zero star formation, deriving an upper limit compatible with previous works. This low-density region (both in gas and SFR) lies formally on the Kennicutt-Schmidt law.

The circum-nuclear region of M 31 appears depleted in gas, both $\mathrm{HI}$ and $\mathrm{H}_{2}$. This depletion is compatible with the absence of star formation. The gas and dust observed in this region are heated in the centre by the radiation of intermediate and old stellar populations. The galaxy then appears to be quenched from inside out, since star formation continues to occur in the outer ring. The presence of diffuse ionised gas in the centre, together with low abundance of neutral gas (atomic and molecular) and lack of young stars in the centre is quite similar to the LIER galaxies described by Belfiore et al. (2016).

In the case of M31, many scenarios have been proposed. Dong et al. (2018) observations have suggested that there was a strong peak in SFR less than $500 \mathrm{Myr}$ ago, which could contribute to the gas depletion. This is in line with the conclusions of Block et al. (2006), which point at a collision with M 32 as a reason for the low molecular gas density in the centre of Andromeda, also explaining the atypical two-ring architecture of M31. D'Souza \& Bell (2018) have claimed that M32 was in the past a much bigger galaxy that was able to produce a major interaction with M312 Gyr ago. This theory also allows the remaining M32-core to have re-entered the M31 disc almost head-on towards the centre and more recently produce the two rings in the disc. Observations of higher J CO emission, and also shocktracing molecules will be helpful to disentangle the various scenarios.

Acknowledgements. Based on observations carried out with the IRAM Plateau de Bure Interferometer. IRAM is supported by INSU/CNRS (France), MPG (Germany), and IGN (Spain). We acknowledge the IRAM Plateau de Bure team for the observations. We thank Sabine König for her support in the data reduction. We warmly thank Eric Rosolowsky, our referee, for his very helpful an constructive comments. We are grateful to Pauline Barmby for providing us with reduced Spitzer data in the nucleus region. ALM has benefited from the support of Action Fédératrice Structuration de l'Univers et Cosmologie from Paris Observatory. This research has made use of the NASA/IPAC Infrared Science Archive, which is operated by the Jet Propulsion Laboratory, California Institute of Technology, under contract with the National Aeronautics and Space Administration. This research made use of Montage, funded by the National Aeronautics and Space Administration's Earth Science Technology Office, Computational Technnologies Project, under Cooperative Agreement Number NCC5 626 between NASA and the California Institute of Technology. The code is maintained by the NASA/IPAC Infrared Science Archive. This research makes use of GALEX, SDSS, 2MASS, Spitzer, and WISE archive data of Andromeda.

\section{References}

Aniano, G., Draine, B. T., Gordon, K. D., \& Sandstrom, K. 2011, PASP, 123, 1218

Baldry, I., Balogh, M., Glazebrook, K., et al. 2006, MNRAS, 373, 469

Barnes, J. E., \& Hernquist, L. E. 1991, ApJ, 370, L65

Begelman, M. C., Volonteri, M., \& Rees, M. J. 2006, MNRAS, 370, 289

Belfiore, F., Maiolino, R., Maraston, C., et al. 2016, MNRAS, 461, 3111

Bertoldi, F., \& McKee, C. F. 1992, ApJ, 395, 140

Bigiel, F., Leroy, A., Walter, F., et al. 2008, AJ, 136, 2846

Bigiel, F., Leroy, A., Walter, F., et al. 2010, AJ, 140, 1194

Block, D. L., Bournaud, F., Combes, F., et al. 2006, Nature, 443, 832

Bolatto, A. D., Wolfire, M., \& Leroy, A. K. 2013, ARA\&A, 51, 207

Boulesteix, J., Georgelin, Y. P., Lecoarer, E., et al. 1987, A\&A, 178, 91

Bower, R. G., Schaye, J., Frenk, C. S., et al. 2017, MNRAS, 465, 32

Braun, R., Thilker, D. A., Walterbos, R. A. M., \& Corbelli, E. 2009, ApJ, 695, 937

Bressan, A., Granato, G. L., \& Silva, L. 1998, A\&A, 332, 135

Caldù-Primo, A., \& Schruba, A. 2016, AJ, 151, 34

Calzetti, D., Liu, G., \& Koda, J. 2012, ApJ, 752, 98

Chang, P., Murray-Clay, R., Chiang, E., \& Quataert, E. 2007, ApJ, 668, 236

Corbelli, E., Braine, J., Bandiera, R., Brouillet, N., et al. 2017, A\&A, 601, A146
Corsini, E. M., Mendez-Abreu, J., Pastorello, N., et al. 2012, MNRAS, 423, L79

Crane, P. C., Dickel, J. R., \& Cowan, J. J. 1992, ApJ, 390, L9

Croton, D. J., Springel, V., White, S. D. M., et al. 2006, MNRAS, 365, 11

Dalcanton, J. J., Williams, B. F., Lang, D., et al. 2012, ApJS, 200, 18

Davis, T. A., Young, L. M., Crocker, A. F., et al. 2014, MNRAS, 444, 342

Di Matteo, T., Springel, V., \& Hernquist, L. 2005, Nature, 433, 604

Dong, H., Olsen, K., Lauer, T., et al. 2018, MNRAS, 478, 5379

Draine, B. T., Aniano, G., Krause, O., et al. 2014, ApJ, 780, 172

D’Souza, R., \& Bell, E. 2018, Nat. Astron., 2, 737

Fabian, A. C. 2012, ARA\&A, 50, 455

Ferrarese, L., \& Merritt, D. 2000, ApJ, 539, L9

Fioc, M., \& Rocca-Volmerange, B. 1999, ArXiv e-prints

[arXiv:astro-ph/9912179]

Fitzpatrick, E. L. 1999, PASP, 111, 63

Fluetsch, A., Maiolino, R., Carniani, S., et al. 2019, MNRAS, 483, 4586

Ford, G. P., Gear, W. K., Smith, M. W. L., et al. 2013, ApJ, 769, 55

Galliano, F., Galametz, M., \& Jones, A. P. 2018, ARA\&A, 56, 673

García-Burillo, S., Combes, F., Schinnerer, E., Boone, F., \& Hunt, L. K. 2005, A\&A, 441, 1011

Gil de Paz, A., Boissier, S., Madore, B. F., et al. 2007, ApJS, 173, 185

Gordon, K. D., Bailin, J., Engelbracht, C. W., et al. 2006, ApJ, 638, L87

Gratier, P., Braine, J., Rodriguez-Fernandez, N. J., et al. 2012, A\&A, 542, A108

Groves, B., Krause, O., Sandstrom, K., et al. 2012, MNRAS, 426, 892

Gueth, F., Guilloteau, S., \& Viallefond, F. 1995, The XXVIIth Young European Radio Astronomers Conference, 8

Gullieuszik, M., Poggianti, B. M., Moretti, A., et al. 2017, ApJ, 846, 27

Gültekin, K., Richstone, D. O., Gebhardt, K., et al. 2009, ApJ, 698, 198

Hammer, F., Yang, Y. B., Wang, J. L., et al. 2018, MNRAS, 475, 2754

Heckman, T. M., \& Best, P. N. 2014, ARA\&A, 52, 589

Heckman, T. M., Kauffmann, G., Brinchmann, J., et al. 2004, ApJ, 613, 109

Hemachandra, D., Barmby, P., Peeters, E., et al. 2015, MNRAS, 454, 818

Hopkins, P. F., \& Quataert, E. 2010, MNRAS, 407, 1529

Hopkins, P. F., Hernquist, L., Cox, T. J., et al. 2006, ApJS, 163, 1

Hughes, A., Meidt, S., Colombo, D., et al. 2013, ApJ, 779, 46

Ibata, R., Irwin, M., Lewis, G., Ferguson, A. M. N., \& Tanvir, N. 2001, Nature, 412, 49

Ibata, R. A., Lewis, G. F., McConnachie, A. W., et al. 2014, ApJ, 780, 128

Inami, H., Armus, L., Charmandaris, V., et al. 2013, ApJ, 777, 156

Jarrett, T. H., Chester, T., Cutri, R., Schneider, S. E., \& Huchra, J. P. 2003, AJ, 125,525

Kaneda, H., Onaka, T., \& Sakon, I. 2005, ApJ, 632, L83

Kauffmann, G., Heckman, M., White, S., et al. 2003, MNRAS, 341, 54

Kennicutt, R. C. 1998, ApJ, 498, 541

King, A., \& Pounds, K. 2015, ARA\&A, 53, 115

Koda, J., Sawada, T., Hasegawa, T., et al. 2006, ApJS, 638, 191

Kormendy, J., \& Ho, L. C. 2013, ARA\&A, 51, 511

Krabbe, A., Sternberg, A., \& Genzel, R. 1994, ApJ, 425, 72

Kruijssen, J. M. D., Longmore, S. N., Elmegreen, B. G., et al. 2014, MNRAS, 440, 3370

Krumholz, M. R., \& McKee, C. F. 2005, ApJ, 630, 250

Lauer, T. R., Bender, R., Kormendy, J., Rosenfield, P., \& Green, R. F. 2012, ApJ, 745,121

Leroy, A. K., Walter, F., Brinks, E., et al. 2008, AJ, 136, 2782

Leroy, A. K., Walter, F., Sandstrom, K., et al. 2013, AJ, 146, 19

Lewis, A. R., Dolphin, A. E., Dalcanton, J. J., et al. 2015, ApJ, 805, 183

Lewis, A. R., Simones, J. E., Johnson, B. D., et al. 2017, ApJ, 834, 70

Li, Z., Garcia, M. R., Forman, W. R., et al. 2011, ApJ, 728, L10

Li, Z., Li, Z., Zhou, P., et al. 2019, MNRAS, 484, 964

Lynden-Bell, D. 1969, Nature, 223, 690

Marconi, A., \& Hunt, L. K. 2003, ApJ, 589, L21

McConnachie, A. W., Irwin, M. J., Ibata, R. A., et al. 2009, Nature, 461, 66

McConnell, N. J., \& Ma, C.-P. 2013, ApJ, 764, 184

McKee, C. F., \& Zweibel, E. G. 1992, ApJ, 399, 551

Melchior, A.-L., \& Combes, F. 2011, A\&A, 536, A52

Melchior, A.-L., \& Combes, F. 2013, A\&A, 549, A27

Melchior, A.-L., \& Combes, F. 2016, A\&A, 585, A44

Melchior, A.-L., \& Combes, F. 2017, A\&A, 607, A7

Melchior, A.-L., Viallefond, F., Guélin, M., \& Neininger, N. 2000, MNRAS, 312 , L29

Miki, Y., Mori, M., \& Rich, R. M. 2016, ApJ, 827, 82

Miville-Deschênes, M.-A., Murray, N., \& Lee, E. J. 2017, ApJ, 834, 57

Molinari, S., Bally, J., Noriega-Crespo, A., et al. 2011, ApJ, 735, L33

Morris, M., \& Serabyn, E. 1996, ARA\&A, 34, 645

Oka, T., Hasegawa, T., Handa, T., Hayashi, M., \& Sakamoto, S. 1996, ApJ, 460, 334

Panuzzo, P., Rampazzo, R., Bressan, A., et al. 2011, A\&A, 528, A10

Peng, Y., Maiolino, R., \& Cochrane, R. 2015, Nature, 521, 192 
Pfenniger, D., \& Norman, C. 1990, ApJ, 363, 391

Pierce-Price, D., Richer, J. S., Greaves, J. S., et al. 2000, ApJ, 545, L121

Poggianti, B. M., Moretti, A., Gullieuszik, M., et al. 2017, ApJ, 844, 48

Rampazzo, R., Panuzzo, P., Vega, O., et al. 2013, MNRAS, 432, 374

Rosenfield, P., Johnson, L. C., Girardi, L., et al. 2012, ApJ, 755, 131

Rosolowsky, E. 2007, ApJ, 654, 240

Rosolowsky, E., \& Leroy, A. 2006, PASP, 118, 590

Saglia, R. P., Fabricius, M., Bender, R., et al. 2010, A\&A, 509, A61

Salak, D., Nakai, N., Hatakeyama, T., \& Miyamoto, Y. 2016, ApJ, 823, 68

Salim, S., Rich, R. M., Charlot, S., et al. 2007, ApJS, 173, 267

Salomé, Q., Salomé, P., Miville-Deschênes, M.-A., Combes, F., \& Hamer, S. 2017, A\&A, 608, A98

Sheth, K., Vogel, S. N., Wilson, C. D., \& Dame, T. M. 2000, Proceedings 232. WE-Heraeus Seminar, 37

Sijacki, D., Vogelsberger, M., Genel, S., et al. 2015, MNRAS, 452, 575

Silk, J., \& Rees, M. J. 1998, A\&A, 331, L1
Solomon, P. M., Rivolo, A. R., Barrett, J., \& Yahil, A. 1987, ApJ, 319, 730 Solomon, P. M., Downes, D., Radford, S. J. E., \& Barrett, J. 1997, ApJ, 478, 144 Soltan, A. 1982, MNRAS, 200, 115

Springel, V., Di Matteo, T., \& Hernquist, L. 2005, MNRAS, 361, 776

Tacconi, L. J., Genzel, R., Neri, R., Cox, P., et al. 2010, Nature, 463, 781

Tenjes, P., Tuvikene, T., Tamm, A., Kipper, R., \& Tempel, E. 2017, A\&A, 600, A34

Thilker, D. A., Braun, R., Walterbos, R. A. M., et al. 2004, ApJ, 601, L39

Tremaine, S., Gebhardt, K., Bender, R., et al. 2002, ApJ, 574, 740

Urry, C. M., \& Padovani, P. 1995, PASP, 107, 803

van der Tak, F. F. S., Black, J. H., Schöier, F. L., Jansen, D. J., \& van Dishoeck, E. F. 2007, A\&A, 468, 627

Viaene, S., Fritz, J., Baes, M., et al. 2014, A\&A, 567, A71

Vega, O., Bressan, A., Panuzzo, P., et al. 2010, ApJ, 721, 1090

Vulic, N., Gallagher, S. C., \& Barmby, P. 2016, MNRAS, 461, 3443

Williams, T. G., Gear, W. K., \& Smith, M. 1. 2018, MNRAS, 479, 297 


\section{Appendix A: Upper limit on the continuum level}

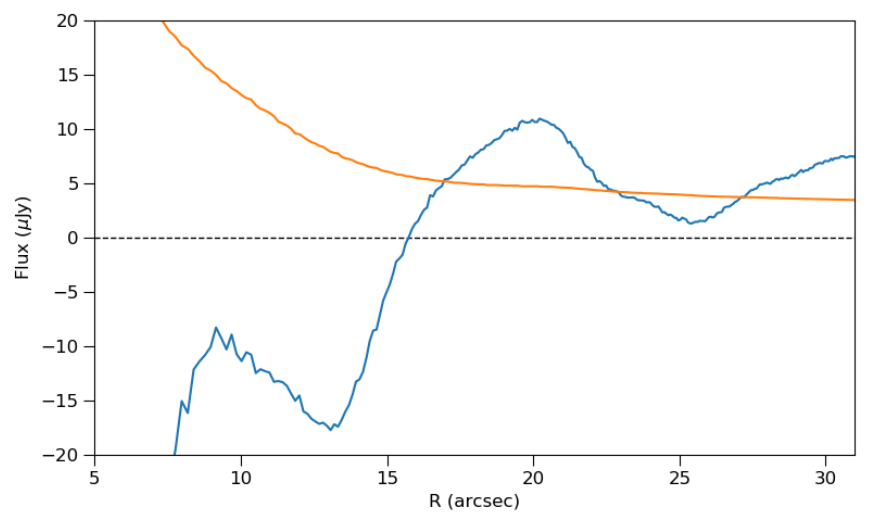

Fig. A.1. Mean flux (blue line) and associated noise (orange line) in $\mu \mathrm{Jy}$ of the spectrum integrated within concentric circles of increasing radius R. Circles are centred on the centre of the field; the channels within the $[-600,0] \mathrm{km} \mathrm{s}^{-1}$ range were removed to avoid influence of the detected clumps.

We estimate an upper limit on the continuum level with an average on the whole bandwidth $[-3000,6000] \mathrm{km} \mathrm{s}^{-1}$. In Fig. A.1, we show the mean flux and associated (rms) noise in $\mu \mathrm{Jy}$ achieved in integrating the whole spectra in circular regions of given radius $\mathrm{R}$. We significantly improve the first $3 \sigma$ upper limit measured at $1 \mathrm{~mm}$ by Melchior \& Combes (2013) of $0.65 \mathrm{mJy}$ with single dish observations. We estimate a $3 \sigma$ upper limit at $3 \mathrm{~mm}$ of about $15 \mu \mathrm{Jy}$ within a radius of $20 \mathrm{arcsec}$.

This excludes the value $(60 \mu \mathrm{Jy})$ at $12 \sigma$ expected with a simple extrapolation of the synchrotron emission detected at $6 \mathrm{~cm}$ $(5 \mathrm{GHz})$ of about $0.2 \mathrm{mJy}$ by Giessubel \& Beck (2014) with a measured spectral index of $-0.4 \pm 0.03$. As the primary beam has a HBPW of 45 arcsec, we do not expect to significantly smooth large scale signals. We thus expect a steeper slope.

We can also exclude large quantities of hidden cold dust. However, it is already well known that this region hosts very little dust (e.g. Melchior et al. 2000). Our upper limit does not provide more constraints than the infrared dust emission presented in the Fig. 3 of Groves et al. (2012), as dust emission at $3 \mathrm{~mm}$ is expected at the level of $10^{-6} \mu \mathrm{Jy}$. If a weak $3 \mathrm{~mm}$ continuum exists in this region, it is probably due to synchrotron emission.

\section{Appendix B: Selection procedure}

With a 2D Gaussian model using the transfer function of a point-like signal we show that isolated pixels showing an excess are impossible to distinguish between signal and noise. We see that a minimum of 3 adjacent spatial pixels is required. We
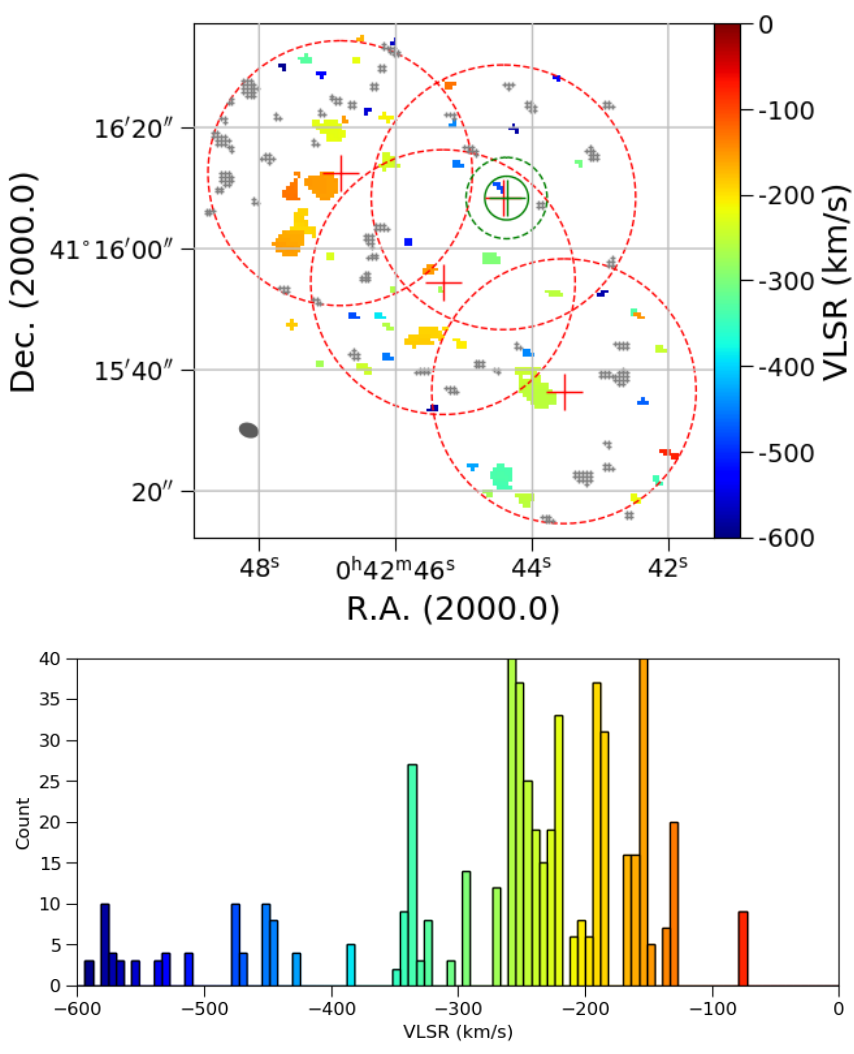

Fig. B.1. Maps of the velocities core clumps detected (top) and associated velocity distribution (down). The colour-coding for the velocity is the same for the maps and histograms. The velocity values for core clumps with contiguous pixels with more than 2 spectral channels above $3 \sigma_{\text {noise }}$ are shown. Positions of the negative core clumps are indicated as grey crosses. The histograms of velocity reveal some spatial and spectral correspondences. Three core clumps with strong signal in particular can be observed at velocities $-157,-187$, and $-253 \mathrm{~km} \mathrm{~s}^{-1}$. This is used to apply GiLDAs cleaning algorithm on specific channels. Some cores are detected outside of the fields and a few also display abnormal velocities for the region studied (especially one at $-76 \mathrm{~km} \mathrm{~s}^{-1}$ ). All these clumps are rejected by our selection procedure.

define as a cloud an area of at least three adjacent spatial pixels with signals with velocities $V_{0}$ varying within a range of $10 \mathrm{~km} \mathrm{~s}^{-1}$ maximum. We find 54 molecular clouds (shown in Fig. B.1) with peak flux ranging from $\Phi_{\max }=11.8 \mathrm{mJy}$ beam $^{-1}$ to $\Phi_{\max }=60.1 \mathrm{mJy} \mathrm{beam}^{-1}$. We also found $3 \sigma$ clumps with negative flux; we represent 47 negative clumps in Fig. B.2. These negative signals are expected to be noise or side lobes: we keep them to test our selection procedure. The velocity distribution histograms for positive and negative clumps show that negative clumps with numerous pixels lie in the same regions as positive core clumps with high $\mathrm{S} / \mathrm{N}$. 

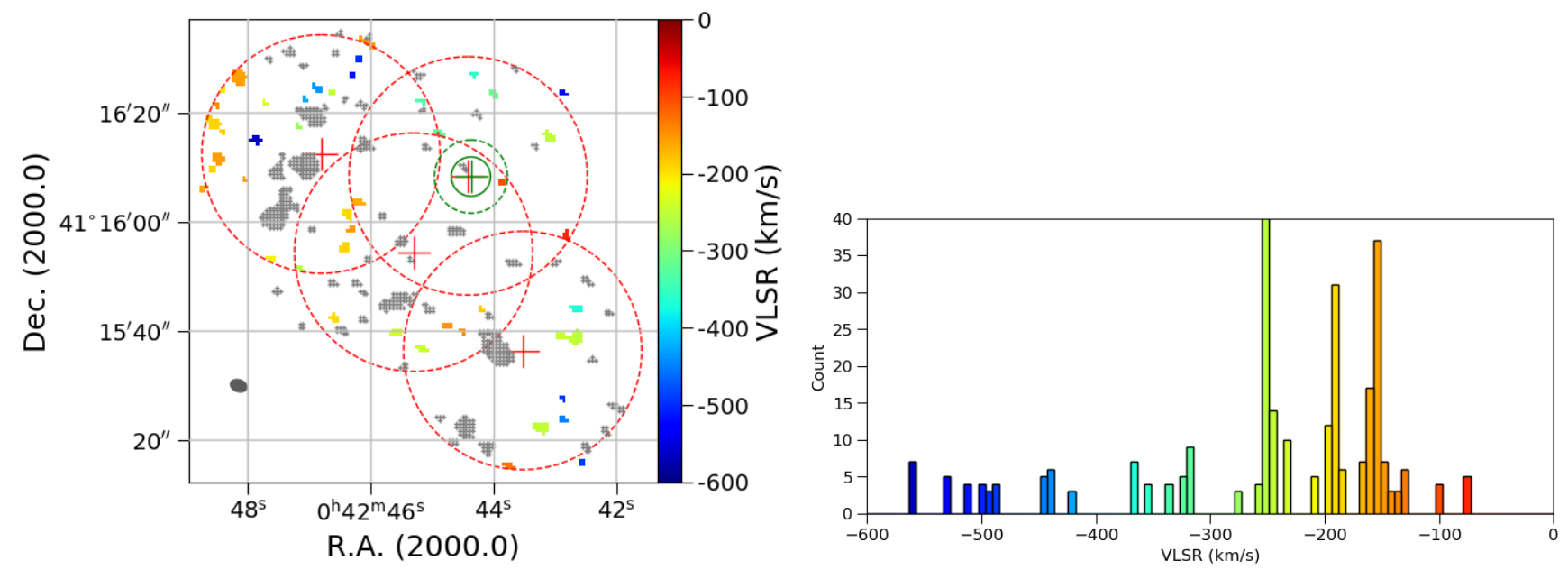

Fig. B.2. Same as Fig. B.1 with the velocity values for core clumps with contiguous pixels with more than 2 spectral channels below $3 \sigma_{\text {noise }}$. Positions of the positive core clumps are shown as grey crosses.
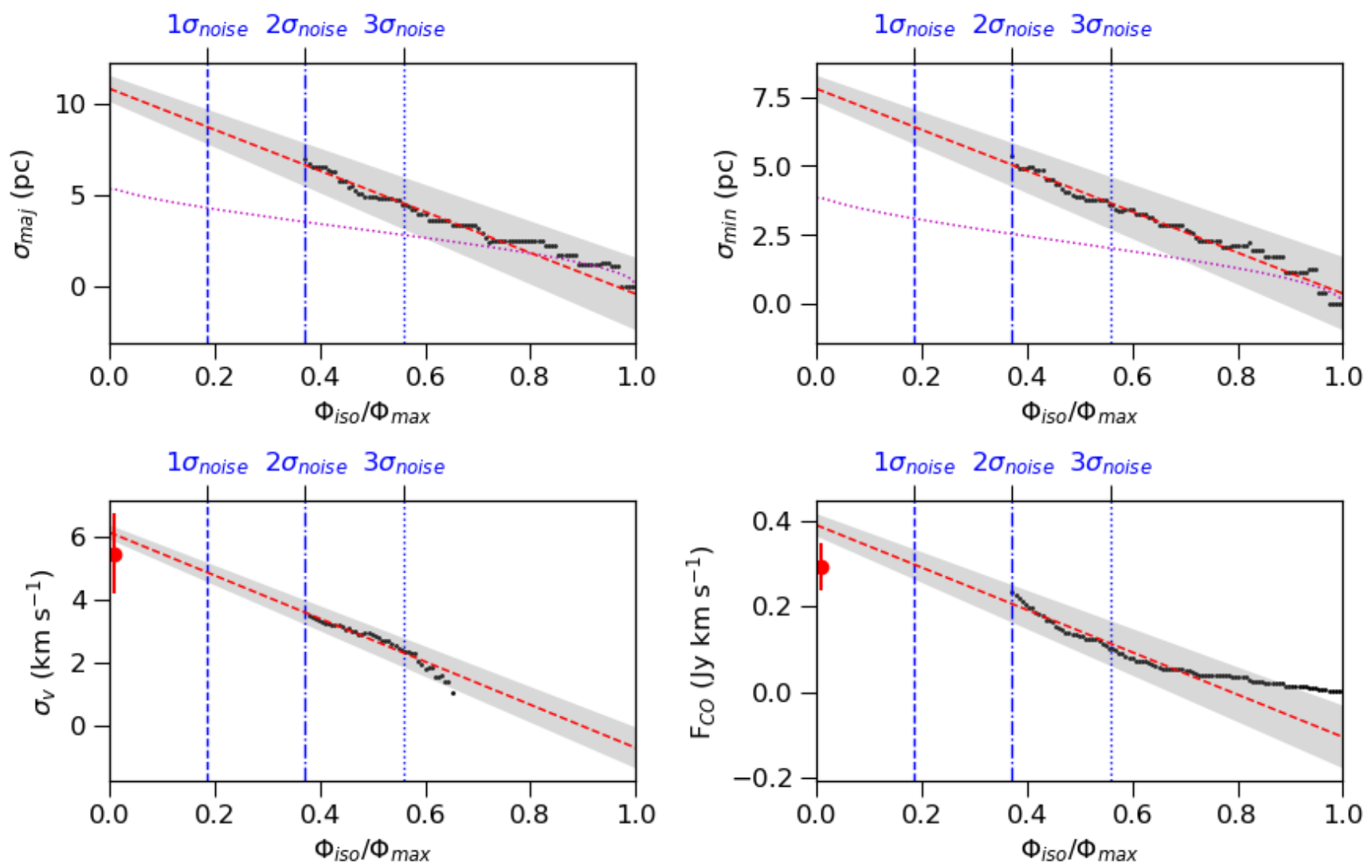

Fig. B.3. Same as Fig. 3 for one clump at $\mathrm{J} 2000$ coordinates $00^{\mathrm{h}} 42^{\mathrm{m}} 44.52^{\mathrm{s}}$ and $+41^{\circ} 16^{\prime} 10.1^{\prime \prime}$ with $S N R_{\text {peak }}=5.4$ and $S N R_{\text {tot }}=15.2$. The size over the major and minor axis is twice as high as that obtained from the simulated clump, which suggests this clump is resolved. The velocity dispersion is more than twice the spectral resolution $\left(2.2 \mathrm{~km} \mathrm{~s}^{-1}\right)$. There is also a good agreement between the extrapolated velocity dispersions, and those measured directly from the spectra, while the total flux is slightly higher for the extrapolation. 